DRAFT VERSION NOVEMBER 9, 2018

Preprint typeset using LTEX style emulateapj v. 03/07/07

\title{
Radio Monitoring of the Tidal DisRuption Event Swift J164449.3+573451. I. Jet EnERgetics and the Pristine Parsec-Scale EnVironment of a Supermassive Black Hole
}

\author{
E. Berger ${ }^{1}$, A. Zauderer ${ }^{1}$, G. G. Pooley ${ }^{2}$, A. M. Soderberg ${ }^{1}$, R. SAri ${ }^{1,3}$, A. Brunthaler ${ }^{4,5}$, And M. F. Bietenholz ${ }^{6,7}$ \\ Draft version November 9, 2018
}

\begin{abstract}
We present continued radio observations of the tidal disruption event Swift J164449.3+573451 extending to $\delta t \approx 216$ days after discovery. The data were obtained with the EVLA, AMI Large Array, CARMA, the SMA, and the VLBA+Effelsberg as part of a long-term program to monitor the expansion and energy scale of the relativistic outflow, and to trace the parsec-scale environment around a previously-dormant supermassive black hole $(\mathrm{SMBH})$. The new observations reveal a significant change in the radio evolution starting at $\delta t \approx 1$ month, with a brightening at all frequencies that requires an increase in the energy by about an order of magnitude, and an overall density profile around the SMBH of $\rho \propto r^{-3 / 2}(0.1-1.2 \mathrm{pc})$ with a significant flattening at $r \approx 0.4-0.6 \mathrm{pc}$. The increase in energy cannot be explained with continuous injection from an $L \propto t^{-5 / 3}$ tail, which is observed in the X-rays. Instead, we conclude that the relativistic jet was launched with a wide range of Lorentz factors, obeying $E\left(>\Gamma_{j}\right) \propto \Gamma_{j}^{-2.5}$. The similar ratio of duration to dynamical timescale for Sw $1644+57$ and GRBs suggests that this result may be applicable to GRB jets as well. The radial density profile may be indicative of Bondi accretion, with the inferred flattening at $r \sim 0.5 \mathrm{pc}$ in good agreement with the Bondi radius for $\mathrm{a} \sim$ few $\times 10^{6} \mathrm{M}_{\odot}$ black hole. The density at $\sim 0.5 \mathrm{pc}$ is about a factor of 30 times lower than inferred for the Milky Way galactic center, potentially due to a smaller number of mass-shedding massive stars. From our latest observations $(\delta t \approx 216 \mathrm{~d})$ we find that the jet energy is $E_{\mathrm{j} \text {,iso }} \approx 5 \times 10^{53} \mathrm{erg}\left(E_{j} \approx 2.4 \times 10^{51} \mathrm{erg}\right.$ for $\theta_{j}=0.1$ ), the radius is $r \approx 1.2 \mathrm{pc}$, the Lorentz factor is $\Gamma_{j} \approx 2.2$, the ambient density is $n \approx 0.2 \mathrm{~cm}^{-3}$, and the projected angular size is $r_{\text {proj }} \approx 25 \mu$ as, below the resolution of the VLBA+Effelsberg. Assuming no future changes in the observed evolution and a final integrated total energy of $E_{j} \approx 10^{52} \mathrm{erg}$, we predict that the radio emission from Sw 1644+57 should be detectable with the EVLA for several decades, and will be resolvable with VLBI in a few years.
\end{abstract}

Subject headings:

\section{INTRODUCTION}

The discovery of the unusual $\gamma$-ray/X-ray transient Swift J164449.3+573451 (hereafter, Sw 1644+57), which coincided with the nucleus of an inactive galaxy at $z=0.354$, has opened a new window into high-energy transient phenomena, with potential implications to our understanding of relativistic outflows in systems such as gamma-ray bursts (GRBs) and active galactic nuclei (AGN). The prevailing interpretation for this event is the tidal disruption of a star by a dormant supermassive black hole $(\mathrm{SMBH})$ with a mass of $M_{\mathrm{BH}} \sim 10^{6}-$ $10^{7} \mathrm{M}_{\odot}$ (Bloom et al. 2011; Burrows et al. 2011; Levan et al. 2011; Zauderer et al. 2011; but see Krolik \& Piran 2011; Ouyed et al. 2011; Quataert \& Kasen 2011 for alternative explanations). The argument for a tidal disruption origin is based on: (i) a positional coincidence $(\lesssim 0.2 \mathrm{kpc})$ with the host galaxy nucleus; (ii) rapid time variability in $\gamma$-rays and X-rays $\left(\lesssim 10^{2} \mathrm{~s}\right)$, which requires a compact source of $\lesssim 0.15$ $\mathrm{AU}$, a few times the Schwarzschild radius of a $\sim 10^{6} \mathrm{M}_{\odot}$

\footnotetext{
${ }^{1}$ Harvard-Smithsonian Center for Astrophysics, 60 Garden Street, Cambridge, MA 02138

${ }^{2}$ Mullard Radio Observatory, Cavendish Laboratory, Cambridge, CB3 OHE UK

${ }^{3}$ Racah Institute of Physics, The Hebrew University, 91904 Jerusalem, Israel

${ }^{4}$ Max-Planck-Institut für Radioastronomie, Auf dem Hügel 69, 53121 Bonn, Germany

${ }^{5}$ National Radio Astronomy Observatory, P.O. Box 0, Socorro, NM 87801

${ }^{6}$ Department of Physics and Astronomy, York University, Toronto, Ontario, Canada

${ }^{7}$ Hartebeesthoek Radio Astronomy Observatory, PO Box 443, Krugersdorp, 1740 South Africa
}

black hole; (iii) high $\gamma$-ray and X-ray luminosity of $\sim 10^{47}$ $\operatorname{erg~s}^{-1}$, which exceeds the Eddington limit of $\mathrm{a} \sim 10^{6} \mathrm{M}_{\odot}$ black hole by $2-3$ orders of magnitude; (iv) a lack of previous radio to $\gamma$-ray activity from this source to much deeper limits than the observed outburst, pointing to a rapid onset; and (v) long-term $X$-ray luminosity evolution following $L_{X} \propto t^{-5 / 3}$, as expected from the fallback of tidally disrupted material (e.g., Rees 1988; Strubbe \& Quataert 2009).

Equally important, Sw $1644+57$ was accompanied by bright radio synchrotron emission, with an initial peak in the millimeter band $\left(F_{\nu} \approx 35 \mathrm{mJy}\right)$ and a steep spectral slope at lower frequencies indicative of self-absorption (Zauderer et al. 2011; hereafter, ZBS11). The properties of the radio emission established the existence of a relativistic outflow with a Lorentz factor of $\Gamma \sim$ few (ZBS11, Bloom et al. 2011). The spectral energy distribution also demonstrated that the lack of detected optical variability required significant rest-frame extinction $\left(A_{V} \gtrsim 5\right.$ mag; ZBS11, Levan et al. 2011), and that the X-rays were produced by a distinct emission component, rather than inverse Compton scattering by the radio-emitting relativistic electrons (ZBS11). Finally, the evolution of the radio emission on a timescale of $\delta t \sim 5-22 \mathrm{~d}$ pointed to an ambient density with a radial profile of roughly $\rho \propto r^{-2}$, as well as a mild increase in the energy of the outflow (ZBS11).

The formation of a relativistic jet with dominant X-ray and radio emission were not predicted in standard tidal disruption models (e.g., Rees 1988; Strubbe \& Quataert 2009), which instead focused on the thermal optical/UV emission from the long-term accretion of the stellar debris. A signature of the 
latter process is a mass accretion rate that evolves as $\dot{M} \propto$ $t^{-5 / 3}$, presumably leading to emission with the same temporal dependence (e.g., Komossa \& Greiner 1999; Gezari et al. 2008; van Velzen et al. 2011). Shortly before the discovery of Sw 1644+57, Giannios \& Metzger (2011) investigated the potential signature of a putative relativistic outflow, and concluded that the interaction of the outflow with the ambient medium will lead to radio emission on a timescale of $\delta t \sim 1$ yr (for typical off-axis observers). While the mechanism for the radio emission from $\mathrm{Sw} 1644+57$ is interaction with an external medium, the actual light curves differ from the offaxis prediction. To address this issue, in a follow-up paper Metzger et al. (2011) (hereafter, MGM11) reconsidered the model for a relativistic jet interacting with an ambient medium. They draw on the inferences from the early radio emission described in ZBS11 to infer the properties of the environment and the jet kinetic energy, and use this information to predict the future evolution of the radio emission.

This long-term radio evolution is of great interest because it can provide several critical insights:

- The integrated energy release in the relativistic outflow, including the anticipated injection from on-going accretion.

- The density profile around a previously-dormant SMBH on $\sim 0.1-10$ pc scales, which cannot be otherwise probed in AGN.

- The potential to spatially resolve the outflow with very long baseline interferometry (VLBI), and hence to measure the dynamical evolution (expansion and potentially spreading) of a relativistic jet.

- Predictions for the radio emission from tidal disruption jets as viewed by off-axis observers on timescales of months to years to decades.

The energy scale and jet dynamics are of particular importance since the total energy input and the structure of the jet may also have implications for relativistic jets in GRBs and AGN. The ability to trace the environment on parsec scales provides a unique probe of gas inflow or outflow around an inactive SMBH on scales that cannot be probed outside of the Milky Way. Finally, the long-term radio emission from Sw 1644+57 will inform future radio searches for tidal disruption events (TDEs) that can overcome the low detection rate in $\gamma$-rays/X-rays (due to beaming), and obscuration due to extinction in the optical/UV (as in the case of Sw 1644+57).

To extract these critical properties we are undertaking longterm monitoring of the radio emission from Sw 1644+57 using a wide range of centimeter- and millimeter-band facilities. Here we present radio observations of Sw 1644+57 that extend to $\delta t \approx 216 \mathrm{~d}$, and use these observations to determine the evolution of the total energy and ambient density. We find that the evolution of both quantities deviates from the behavior at $\delta t \lesssim 1$ month (presented in ZBS11), thereby providing crucial insight into the structure of the relativistic outflow and the ambient medium. This paper is the first in a series that will investigate the long-term radio evolution of Sw 1644+57 and the implications for relativistic jets and parsec-scale environments around supermassive black holes, including efforts to resolve the source with VLBI and to measure polarization.

The current paper is organized as follows. We describe the radio observations in $\$ 2$, and summarize the radio evolution at $\delta t \approx 5-216 \mathrm{~d}$ in $\$ 3$. In $\$ 4$ we present our modeling of the radio emission, which utilizes the formulation of MGM11. The implications for the energy scale and ambient density are discussed in $\$ 4.1$ and $\$ 4.2$, respectively, and we finally consider the implications for relativistic jets and the parsec-scale environments of SMBHs in $\$ 5$.

\section{RADIO OBSERVATIONS}

Although Sw 1644+57 first triggered the Swift Burst Alert Telescope on 2011 March 28.55 UT, discernible $\gamma$-ray emission was detected starting on 2011 March 25 UT (Burrows et al. 2011). We therefore consider 2011 March 25.5 UT to be the actual initial time for the event and the associated relativistic outflow that powers the observed radio emission. Our radio observations of $\mathrm{Sw} 1644+57$ commenced on 2011 March 29.36 UT (19.4 hr after the Swift trigger and about $3.9 \mathrm{~d}$ after the initial $\gamma$-ray detection). Observations extending to $\delta t \approx 26 \mathrm{~d}$ were presented in ZBS11. Here we report observations extending to $\delta t \approx 216 \mathrm{~d}$. Throughout the paper we use the standard cosmological constants with $H_{0}=70 \mathrm{~km}$ $\mathrm{s}^{-1} \mathrm{Mpc}^{-1}, \Omega_{m}=0.27$ and $\Omega_{\Lambda}=0.73$.

\subsection{Expanded Very Large Array}

We observed Sw 1644+57 with the EVLA ${ }^{8}$ using the new Wideband Interferometric Digital Architecture (WIDAR) correlator to obtain up to $2 \mathrm{GHz}$ of bandwidth at several frequencies. At all frequencies, we used 3C286 for bandpass and flux calibration. At $1.4 \mathrm{GHz}$, we used J1634+6245 for phase calibration. For phase calibration at all other frequencies, we used $\mathrm{J} 1638+5720$, and also included a third calibrator, $\mathrm{J} 1639+5357$, at $5.8 \mathrm{GHz}$. The data were reduced and imaged with the Astronomical Image Processing System (AIPS) software package. The observations are summarized in Table 1 Minor changes to data values with respect to those reported in ZBS11 are due to additional flagging of the data. The errors reported in Table 1 are statistical uncertainties only; the overall systematic uncertainty in the flux calibration is $\lesssim 5 \%$.

\subsection{AMI Large Array}

We observed with the AMI Large Array at $15.4 \mathrm{GHz}$ with a bandwidth of $3.75 \mathrm{GHz}$. The maximum baseline is about 110 $\mathrm{m}$, with a resulting angular resolution of 25 arcsec. Observations ranged in duration from $45 \mathrm{~min}$ to $11 \mathrm{hr}$. Observations of the compact source $\mathrm{J} 1638+5720$ were interleaved at intervals of $10 \mathrm{~min}$ as a phase reference, and the flux density scale was established by regular observations of the calibrators $3 \mathrm{C} 48$ and 3C286. The telescope measures linearly-polarized signals (Stokes I+Q). The observations are summarized in Table 1.

\subsection{Combined Array for Research in Millimeter Astronomy}

We observed Sw 1644+57 with CARMA at frequencies of 87.3 and $93.6 \mathrm{GHz}$ with a total bandwidth ranging between 6.8 and $7.8 \mathrm{GHz}$. We used Neptune as our primary flux calibrator, and $\mathrm{J} 1824+568$ and $\mathrm{J} 1638+573$ as bandpass and phase calibrators. The overall uncertainty in the absolute flux calibration is $\approx 20 \%$. Data calibration and imaging were done with the MIRIAD and AIPS software packages. The observations are summarized in Table 1. We note some changes

\footnotetext{
8 The EVLA is operated by the National Radio Astronomy Observatory, a facility of the National Science Foundation operated under cooperative agreement by Associated Universities, Inc. The observations were obtained as part of programs 10C-145, 11A-262, and 11A-266
} 
with respect to the flux densities reported in ZBS11, especially those epochs where the total integration time on source was less than $\sim 20 \min (\delta t \approx 10-25 \mathrm{~d})$.

\subsection{Submillimeter Array}

We observed Sw 1644+57 with the SMA using at least seven of the eight antennas, in a wide range of weather conditions, with $\tau_{225}$ ranging from 0.04 to 0.3 . In each observation we combined the two sidebands, each with a bandwidth of $4 \mathrm{GHz}$ separated by $10 \mathrm{GHz}$, to increase the signal-to-noise ratio. The data were calibrated using the MIR software package developed at Caltech and modified for the SMA, while for imaging and analysis we used MIRIAD. Gain calibration was performed using J1642+689, 3C345, and J1849+670. Absolute flux calibration was performed using real-time measurements of the system temperatures, with observations of Neptune to set the overall scale. Bandpass calibration was done using 3C454.3, J1924-292, and 3C279. The observations are summarized in Table 1 .

\subsection{Very Long Baseline Interferometry}

We observed Sw 1644+57 with the NRAO Very Long Baseline Array $\left(\mathrm{VLBA}^{9}\right)$ and the 100-m Effelsberg telescope in six epochs ${ }^{10}$ between 2011 April 2 and September 17 UT at 8.4 and $22 \mathrm{GHz}$. The observations were performed with eight frequency bands of $8 \mathrm{MHz}$ bandwidth each in dual circular polarization, resulting in a total data rate of $512 \mathrm{Mbps}$. ICRF J1638+5720 (Ma et al. 1998), located only $0.92^{\circ}$ from Sw 1644+57 was used for phase-referencing at both frequencies. At $22 \mathrm{GHz}$, we switched between the target and calibrator every $40 \mathrm{~s}$, while at $8.4 \mathrm{GHz}$ we spent $40 \mathrm{~s}$ on the calibrator and $90 \mathrm{~s}$ on the target. This resulted in a total integration time on $\mathrm{Sw} 1644+57$ of about $93 \mathrm{~min}$ at $8.4 \mathrm{GHz}$ and $78 \mathrm{~min}$ at $22 \mathrm{GHz}$ in epochs 1, 2, 5, and 6. Epochs 3 and 4 were 1 hour shorter, resulting in integration times on source of $75 \mathrm{~min}$ at $8.4 \mathrm{GHz}$ and $60 \mathrm{~min}$ at $22 \mathrm{GHz}$. A second calibrator, J1657+5705 (Beasley et al. 2002), was observed for 6 min at each frequency to check the overall data calibration. At $22 \mathrm{GHz}$, we also performed $\sim 30$-min blocks of geodetic observations to perform atmospheric calibration (for details see Brunthaler et al. 2005; Reid et al. 2009).

The results from epoch 1 were presented in ZBS11. Here, we only discuss the $22 \mathrm{GHz}$ data; the full VLBI data set will be presented together with future VLBI observations in an upcoming paper. The data were correlated at the VLBA Array Operations Center in Socorro, New Mexico and calibrated using AIPS and ParselTongue (Kettenis et al. 2006). We applied the latest values of the Earth orientation parameters and performed zenith delay corrections based on the results of the geodetic block observations. Total electron content maps of the ionosphere were used to correct for ionospheric phase changes. Amplitude calibration used system temperature measurements and standard gain curves. We performed a "manual phase-calibration" using the data from one scan of J1638+5720 to remove instrumental phase offsets among the frequency bands. We then fringe-fitted the data from $\mathrm{J} 1638+5720$. Since $\mathrm{J} 1638+5720$ has extended structure, we performed phase self-calibration, and later amplitude and phase self-calibration on J1638+5720 to construct robust models of $\mathrm{J} 1638+5720$ at both frequencies. These

\footnotetext{
${ }^{9}$ The data were collected as part of programs BS210 and BS212.

10 The dates of the observations are: 2011 April 2, April 8, May 5, May 28, July 17 , and September 17 UT.
}

models were then used to fringe-fit the data again. Finally, the calibration was transferred to Sw $1644+57$ and J1657+5705. The data were imaged in AIPS using robust weighting (with ROBUST=0).

A linear fit to the positions of Sw $1644+57$ from epochs $2-6$

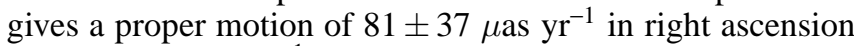
and $46 \pm 59 \mu \mathrm{as} \mathrm{yr}^{-1}$ in declination, with an additional systematic uncertainty of about $40 \mu \mathrm{as} \mathrm{yr}^{-1}$. This is consistent with no detectable motion. The corresponding $3 \sigma$ upper limit on the motion at the redshift of Sw $1644+57$ is $\lesssim 1 \mathrm{pc}$. Since the first epoch was correlated at a different position, it was not included in this fit to avoid second order systematic errors.

To obtain an accurate estimate of the angular size in our latest epoch (2011 September 17 UT), we fit Gaussian models directly to the visibility data. We first self-calibrated the data in phase using solution intervals of $20 \mathrm{~min}$, and then fitted a circular Gaussian model using a weighted least-squares fit. The source is not resolved in our observation. For unresolved sources the fitted size can be strongly correlated with the antenna amplitude gains, which are only imprecisely known. Our uncertainty on the fitted FWHM size, and consequently our upper limit, was estimated from a Monte-Carlo simulation in which we randomly varied the amplitude gains of the antennas by conservative factors of $\pm 25 \%$ (for a more elaborate discussion of the uncertainties in estimating angular source sizes from a similar procedure see Bietenholz et al. 2010). The $3 \sigma$ upper limit on the FWHM source size is 0.22 mas, corresponding to a projected physical diameter of $\lesssim 1.1 \mathrm{pc}$. For spherical expansion this corresponds to an upper limit on the apparent expansion velocity of $\lesssim 3.8 c$. For a collimated relativistic source with an opening angle $\theta_{j}$ the projected diameter is $\approx 4 \Gamma^{2} \operatorname{ct} \theta_{j}$, indicating that for $\theta_{j}=0.1$ the limit on the size of Sw $1644+57$ corresponds to $\Gamma \lesssim 5$ at $\delta t \approx 176 \mathrm{~d}$. We compare this result with estimates of the source size from modeling of the radio emission in $\$ 4.1$.

\section{OBSERVED EVOLUTION OF THE RADIO EMISSION}

The light curves at observed frequencies of 4.9, 6.7, 15.4, 19, 24, 43, 90, and $230 \mathrm{GHz}$ are presented in Figure 1. As described in ZBS11, the light curves at 4.9 to $24 \mathrm{GHz}$ exhibit an initial rapid increase, with a flux density of $F_{\nu} \propto t^{1.5}-t^{2}$, followed by a shallower increase, with $F_{\nu} \propto t^{0.5}$. In the millimeter band, the light curves peak on a timescale of a few days and then decline as $F_{\nu} \propto t^{-1}$. This evolution is due to a synchrotron spectrum that is optically thin in the millimeter band and optically thick in the centimeter band, with a peak frequency and flux density that decline as a function of time as $\nu_{p} \propto t^{-1.3}$ and $F_{\nu, p} \propto t^{-0.8}$ (ZBS11). The properties of the spectral energy distribution, along with measurements of interstellar scintillation, established that the outflow is relativistic $(\Gamma \approx 2-5)$, that the density profile of the circumnuclear medium $(\mathrm{CNM})$ is roughly $\rho_{\mathrm{CNM}} \propto r^{-2}$, and that the post-shock energy increases as $E \propto t^{0.5}$ (ZBS11).

The most striking result from our new observations is that the millimeter flux on a timescale of $\sim 100 \mathrm{~d}$ is significantly brighter than expected from an extrapolation of the early decline. Indeed, at $90 \mathrm{GHz}$ the brightness is comparable to the initial peak at $\delta t \sim 10 \mathrm{~d}$. A similar effect is observed at lower frequencies, where the light curves exhibit an upturn starting at $\approx 30 \mathrm{~d}$, with $F_{\nu} \propto t^{0.7}$. The $90 \mathrm{GHz}$ light curve is already in a declining (i.e., optically thin) phase at $\delta t \gtrsim 100 \mathrm{~d}$, while the light curves at 4.9 to $24 \mathrm{GHz}$ peak at $\delta t \approx 100 \mathrm{~d}$ (19 and $24 \mathrm{GHz}), \delta t \approx 125 \mathrm{~d}(15.4 \mathrm{GHz}), \delta t \approx 170 \mathrm{~d}(8.4 \mathrm{GHz})$, and $\delta t \approx 190 \mathrm{~d}(4.9$ and $6.7 \mathrm{GHz})$, with peak flux densities of $\approx 31$ 
$\mathrm{mJy}, \approx 28 \mathrm{mJy}, \approx 23 \mathrm{mJy}$, and $\approx 20 \mathrm{mJy}$, respectively. These observations are at odds with the light curve evolution predicted by MGM11 based on our radio data at 5-22 d, using a model that assumes a constant energy and a steady $\rho \propto r^{-2}$ CNM profile (Figure 1).

The observed change in the light curve evolution at $\delta t \gtrsim$ 1 month requires an increase in the outflow kinetic energy and/or the CNM density. The evolution of the peak frequency, roughly $\nu_{p} \propto t^{-1}$ at $\delta t \sim 30-100 \mathrm{~d}$, is slower than expected for the characteristic synchrotron frequency, $\nu_{m} \propto t^{-1.5}$ (see \$4). Since $\nu_{m}$ only depends on the kinetic energy, $\nu_{m} \propto E_{j}^{0.5} t^{-3 / 2}$ (Granot \& Sari 2002), the observed evolution requires an increase in the energy, with $E_{j} \propto t$. At the same time, the similar light curve evolution at 4.9 and $24 \mathrm{GHz}$, which straddle the self-absorption frequency (see \$4), indicates that the density must also change. In particular, at $4.9 \mathrm{GHz}$ we expect $F_{\nu} \propto$ $E_{j}^{1.5} A^{-1.5} t^{0.5}$, while at $24 \mathrm{GHz}$ we expect $F_{\nu} \propto E_{j}^{5 / 6} A^{0.5} t^{-0.5}$; here $A$ is a fiducial density that parametrizes the CNM density, with $\rho=A r^{-2}$. For the observed light curve evolution of $F_{\nu} \propto t^{0.7}$ at both frequencies, and using the inferred evolution of $E_{j}$, we find that $A \propto t^{0.85}$. Thus, both the energy and density normalization appear to increase at $\delta t \gtrsim 30 \mathrm{~d}$. In the next section we model the radio emission in detail to extract the temporal evolution of the energy and density.

\section{DETAILED MODELING OF THE RADIO EMISSION}

To determine the temporal evolution of the relativistic outflow and the radial density profile we use the formulation of MGM11, which draws on the GRB afterglow model of Granot \& Sari (2002) (hereafter, GS02). The MGM11 model is largely motivated by the basic properties of the outflow that were determined in our initial study of the radio emission (ZBS11), as well as by the observed evolution of the Xray emission (Burrows et al. 2011). The model assumes that the outflow is collimated, with an opening angle $\theta_{j}$, and has a Lorentz factor of $\Gamma_{i}$; in ZBS11 we demonstrated that $\Gamma_{j} \sim$ few (see also Bloom et al. 2011). The kinetic luminosity of the outflow, $L_{\mathrm{j}, \text { iso }}$, is assumed to be constant for a timescale $t_{j}$, followed by a decline as $L_{\mathrm{j}, \text { iso }} \propto t^{-5 / 3}$ at $t \gtrsim t_{j}$. This is expected for fallback accretion in a TDE, and is observationally motivated by the evolution of the X-ray light curve, which also indicates that $t_{j} \approx 10^{6} \mathrm{~s}$ (Burrows et al. 2011). As a result of the long-term injection of energy, the expectation is that the kinetic energy will gradually increase from an initial value of $E_{\mathrm{j}, \text { iso }}=L_{\mathrm{j}, \text { iso }} t_{j}$ to a final level of $(5 / 2) L_{\mathrm{j}, \text { iso }} t_{j}$. From the initial $\mathrm{X}$-ray luminosity, $L_{\mathrm{X} \text {,iso }} \approx 6 \times 10^{46} \mathrm{erg} \mathrm{s}^{-1}$, the expected total energy is $E_{\mathrm{j} \text {,iso }} \approx 3 \times 10^{53}\left(\epsilon_{X} / 0.5\right)^{-1} \mathrm{erg}$, where $\epsilon_{X}$ is the efficiency of the jet in producing X-rays (MGM11), while the predicted beaming-corrected energy is $E_{j} \approx 1.5 \times 10^{51} \mathrm{erg}$.

The interaction of the outflow with the CNM leads to synchrotron emission due to the acceleration of electrons and amplification of magnetic fields. Based on the early radio observations, ZBS11 demonstrated that the CNM density roughly follows $\rho_{\mathrm{CNM}} \propto r^{-2}$ (hereafter, Wind medium), which is the profile adopted for the analysis in MGM11. The Lorentz factor of the fluid behind the forward shock is $\Gamma_{\mathrm{sh}, \mathrm{FS}}=\Gamma_{j}(1-$ $\left.\Gamma_{j} \sqrt{2 n_{\mathrm{CNM}} / 7 n_{j}}\right)$, where $n_{\mathrm{CNM}}$ is the circumnuclear density and $n_{j}=L_{\mathrm{j}, \text { iso }} / 4 \pi r^{2} m_{p} c^{3} \Gamma_{j}^{2}$ is the density of the ejecta (see MGM11); this relation is appropriate for the case of a Newtonian reverse shock, i.e., $\Gamma_{j}^{2} \ll n_{j} / n_{\mathrm{CNM}}$ (Sari \& Piran 1995), which as we find below is appropriate for $\mathrm{Sw} 1644+57$ (Table 2). A key difference between the MGM11 model and the
GS02 GRB afterglow model is that the former includes a suppression of the flux density by a factor of $\left(\Gamma_{\text {sh,FS }} \theta_{j}\right)^{2} / 2$ due to the finite extent of the collimated outflow (i.e., $\Gamma_{\text {sh,FS }} \lesssim 1 / \theta_{j}$ ). Thus, the synchrotron emission from the relativistic outflow is determined by $L_{\mathrm{j}, \text { iso }}, n_{\mathrm{CNM}}$, and the fractions of post-shock energy in the radiating electrons $\left(\epsilon_{e}\right)$ and the magnetic field $\left(\epsilon_{B}\right)$.

We use this model (Equations 7-11 of MGM11), along with the smoothing formulation of GS02, to fit snap-shot broadband spectra of $\mathrm{Sw} 1644+57$ on a timescale of $\delta t \approx 5-216$ d. In each epoch we fix $\epsilon_{e}=\epsilon_{B}=0.1$ and $p=2.5$, and we then determine the best-fit values of $L_{\mathrm{j}, \text { iso }}$ and $n_{\mathrm{CNM}}$; here $p$ is the power law index of the electron Lorentz factor distribution, $N(\gamma) \propto \gamma^{-p}$. For our choice of $p$ value the synchrotron frequencies (self-absorption: $\nu_{a}$; peak: $\nu_{m}$ ) and flux normalization $\left[F_{\nu}\left(\nu=\nu_{a}\right)\right]$ for $t \gtrsim t_{j}$ are given by (MGM11, GS02):

$$
\begin{gathered}
\nu_{a}(t)=4.2 \times 10^{9} \epsilon_{e,-1}^{-1} \epsilon_{B,-1}^{0.2} L_{\mathrm{j}, \text { iso }, 48}^{-0.4} t_{j, 6}^{-1} n_{18}^{1.2}\left(\frac{t}{t_{j}}\right)^{-0.6} \mathrm{~Hz}(1) \\
\nu_{m}(t)=3.5 \times 10^{11} \epsilon_{e,-1}^{2} \epsilon_{B,-1}^{0.5} L_{\mathrm{j}, \text { iso }, 48}^{0.5} t_{j, 6}^{-1}\left(\frac{t}{t_{j}}\right)^{-1.5} \mathrm{~Hz}, \quad(2) \\
F_{\nu}\left[\nu_{a}(t)\right]=345 \epsilon_{e,-1} L_{\mathrm{j}, \text { iso }, 48}^{1.5} t_{j, 6}^{2} n_{18}^{-1.5} \theta_{j,-1}^{2} \nu_{a, 10}^{2}\left(\frac{t}{t_{j}}\right)^{0.5} \mathrm{mJy}(3)
\end{gathered}
$$

where we use the notation $X \equiv 10^{y} X_{y}$, and $n_{18}$ is the CNM density $\left(n_{\mathrm{CNM}}\right)$ at a fiducial radius of $10^{18} \mathrm{~cm}$. At $t \lesssim t_{j}$ we use the same equations but with the time dependencies modified to $\nu_{a} \propto t^{-1}, \nu_{m} \propto t^{-1}$, and $F_{\nu}\left(\nu=\nu_{a}\right) \propto t^{2}$ (MGM11). The synchrotron spectrum is given by (c.f., Spectrum 1 of Granot \& Sari 2002):

$$
\begin{gathered}
F_{\nu}=F_{\nu}\left(\nu_{a}\right)\left[\left(\frac{\nu}{\nu_{a}}\right)^{-s_{1} \beta_{1}}+\left(\frac{\nu}{\nu_{a}}\right)^{-s_{1} \beta_{2}}\right]^{-1 / s_{1}} \times \\
{\left[1+\left(\frac{\nu}{\nu_{m}}\right)^{s_{2}\left(\beta_{2}-\beta_{3}\right)}\right]^{-1 / s_{2}},}
\end{gathered}
$$

where $s_{1}$ and $s_{2}$ are smoothing parameters (GS02), and $\beta_{1}=2$, $\beta_{2}=1 / 3$, and $\beta_{3}=(1-p) / 2$ are the power law indices for each segment of the synchrotron spectrum.

Equations 1 and 4 are appropriate when $\nu_{a}<\nu_{m}$, and have to be modified when $\nu_{m}<\nu_{a}$ as follows (GSO2, MGM1111):

$$
\begin{gathered}
\nu_{a} \propto t^{-3(p+2) / 2(p+4)}, \\
F_{\nu}=F_{\nu}\left(\nu_{m}\right)\left[\left(\frac{\nu}{\nu_{m}}\right)^{-s_{1} \beta_{1}}+\left(\frac{\nu}{\nu_{m}}\right)^{-s_{1} \beta_{2}}\right]^{-1 / s_{1}} \times \\
{\left[1+\left(\frac{\nu}{\nu_{a}}\right)^{s_{2}\left(\beta_{2}-\beta_{3}\right)}\right]^{-1 / s_{2}},}
\end{gathered}
$$

where the spectrum is now normalized at $\nu=\nu_{m}, s_{1}$ and $s_{2}$, take on different values (GS02) and $\beta_{1}=2, \beta_{2}=5 / 2$, and $\beta_{3}=(1-p) / 2$. To smoothly connect the evolution in the two phases we use a weighted average of Equations 4 and 6 , with the weighting determined by the time difference relative to the transition time, $t_{\mathrm{am}}$, defined as $\nu_{a}\left(t_{\mathrm{am}}\right)=\nu_{m}\left(t_{\mathrm{am}}\right)$. We find

\footnotetext{
11 Note that MGM11 use the scalings appropriate for Spectrum 3 of GS02, while we use the appropriate Spectrum 2 with $\nu_{m}<\nu_{a}<\nu_{c}$.
} 
that $t_{\mathrm{am}} \approx 275 \mathrm{~d}$, so this transition does not affect the data presented in this paper.

Instead of imposing a specific temporal evolution on $L_{\mathrm{j}, \text { iso }}$ and $n_{\mathrm{CNM}}$, we model each broad-band radio spectrum independently to extract the evolution of these quantities, and in turn the time evolution of the emission radius $(r)$ and $\Gamma_{\mathrm{sh}}$ (and hence $\Gamma_{j}$ ), as well as the radial density profile. The results of the individual fits are shown in Figure 2 and the relevant extracted parameters are plotted in Figure 3 and summarized in Table 2 The broad-band SEDs reveal a complex evolution. At $\delta t \approx 5-22 \mathrm{~d}$ both the peak frequency and peak flux density decrease with time (as noted by ZBS11), but starting at $\delta t \approx 36 \mathrm{~d}$ the peak flux density begins to increase, reaching a maximum at $\delta t \approx 100 \mathrm{~d}$, and subsequently declining again.

The VLBI size limit of $r_{\text {proj }} \lesssim 0.55 \mathrm{pc}$ at $\delta t \approx 175 \mathrm{~d}$ can be used to set an independent upper bound on the ratio $L_{\mathrm{j}, \text { iso }, 48} / n_{18}$. In particular, on this timescale we expect $r_{\text {proj }} \approx$ $2.9 t_{j, 6}\left(L_{\mathrm{j}, \text { iso }, 48} / n_{18}\right)^{0.5} \theta_{j}$ pc, indicating that $L_{\mathrm{j}, \text { iso }, 48} / n_{18} \lesssim 3.6$ (for $\theta_{j}=0.1$ ). From the results of our radio modeling we find on a similar timescale that $L_{\mathrm{j} \text {.iso. } 48} \approx 0.45$ and $n_{18} \approx 3$ (Table 21), and hence $L_{\mathrm{j}, \text { iso }, 48} / n_{18} \approx 0.15$ is in agreement with the VLBI size limit. On the other hand, if we assume $\epsilon_{e}=0.01$ the resulting value of $L_{\mathrm{j}, \text { iso }, 48}$ increases by about a factor of 15 , while $n_{18}$ decreases by about a factor of 2 , leading to $L_{\mathrm{j}, \text { iso }, 48} / n_{18} \approx 4$, in violation of the VLBI size limit ${ }^{12} \mathrm{We}$ therefore conclude from the VLBI results that $\epsilon_{e} \gtrsim 0.01$.

\subsection{The Evolution of $E_{\mathrm{j}, \text { iso }}$}

A detailed analysis of the evolution of $\nu_{m}$ (shown in Figure 4) reveals a complex behavior. While it continuously declines as a function of time, the decline rate is always shallower than the expected $t^{-3 / 2}$ for constant energy. Since $\nu_{m}$ only depends on $L_{\mathrm{j}, \text { iso }}$ and the equipartition fractions (which are not expected to change with time), the shallower than expected evolution directly implies that $L_{\mathrm{j}, \text { iso }}$ continuously increases with time. In particular, at $5-22 \mathrm{~d}$ and $\gtrsim 100 \mathrm{~d}$, the observed evolution of $\nu_{m} \propto t^{-1.15}$ indicates that $L_{\mathrm{j}, \text { iso }} \propto t^{0.7}$, while in the intermediate phase $(22-100) \mathrm{d}, \nu_{m} \propto t^{-0.95}$ points to a more rapid increase in the energy scale, with $L_{\mathrm{j}, \text { iso }} \propto t^{1.1}$.

The time evolution of $L_{\mathrm{j}, \text { iso }}$ is shown in Figure 5. We

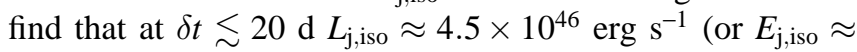
$4.5 \times 10^{52}$ erg for $t_{j}=10^{6} \mathrm{~s}$ ). Changing the values of the equipartition fractions from our assumed values $\epsilon_{e}=\epsilon_{B}=0.1$, we find that $L_{\mathrm{j}, \text { iso }}$ increases by about a factor of 3 if $\epsilon_{B}=0.01$ or by a factor of 15 if $\epsilon_{e}=0.01\left(\epsilon_{e} \lesssim 0.01\right.$ is ruled out by the VLBI limits on the source size). These scalings hold for the overall time evolution of $L_{\mathrm{j} \text { iso }}$.

Also shown in Figure 5 is the expected evolution for a model with $L_{\mathrm{j}, \text { iso }}=$ const at $t<t_{j}$ and $L_{\mathrm{j} \text {.iso }} \propto t^{-5 / 3}$ at $t>t_{j}$, as inferred from the $\mathrm{X}$-ray light curve (Burrows et al. 2011, MGM11). In this model, we expect the integrated value of $L_{\mathrm{j}, \text { iso }}$ (i.e., the total isotropic-equivalent energy) to approach $(5 / 2) L_{\mathrm{j}, \text { iso }}$ as $t \rightarrow \infty$, and to roughly double within $\delta t \sim 5 t_{j} \approx$ $50-60 \mathrm{~d}$. This seems to be the case based on the radio data at $\delta t \lesssim 30 \mathrm{~d}$, but the subsequent rapid increase in energy by about an order of magnitude at $30-216 \mathrm{~d}$ cannot be explained with continued injection from a $t^{-5 / 3}$ tail. On the other hand, the X-ray light curve of Sw 1644+57 (Figure 5) agrees well with this simple luminosity evolution, with $L_{\mathrm{X}, \text { iso }, 0} \approx 6 \times 10^{46}$ $\mathrm{erg} \mathrm{s}^{-1}$ at $t \lesssim 13 \mathrm{~d}$. A comparison to the inferred value of

\footnotetext{
12 Changing to $\epsilon_{B}=0.01$ has almost no effect since both the energy and density increase by about a factor of 3 compared to $\epsilon_{B}=0.1$.
}

$L_{\mathrm{j}, \text { iso, } 0}$ indicates a high efficiency ${ }^{13}$ of producing X-rays of $\epsilon_{X} \approx 0.5$.

Since continuous energy injection from an $L \propto t^{-5 / 3}$ tail cannot explain the inferred rise in integrated kinetic energy, the radio data require a different energy injection mechanism. One possibility is that the outflow has a distribution of Lorentz factors, with increasing energy as a function of decreasing Lorentz factor, $E_{j}\left(>\Gamma_{j}\right) \propto \Gamma_{j}^{1-s}$ (Sari \& Mészáros 2000). In this scenario the energy will increase with time as material with a lower Lorentz factor catches up with decelerated ejecta that initially had a higher Lorentz factor. The continuous injection of energy will also lead to a more rapid increase in radius, with $r \propto t^{(1+s) /(7+s-2 m)}$ (Sari \& Mészáros 2000); here $m$ is the power law index describing the CNM density profile $\left(\rho \propto r^{-m}\right)$. In Figure 3 we plot the inferred radius as a function of time, and find that it follows $r \propto t^{0.6}$, faster than expected in a simple Wind model $\left(r \propto t^{0.5}\right)$. We also find from the density profile that $m \approx 1.5$ (see $\$ 4.2$ ), thereby leading to $s \approx 3.5$. Thus, we expect in this scenario a fairly steep profile for the energy as a function of Lorentz factor of $E\left(>\Gamma_{j}\right) \propto \Gamma_{j}^{-2.5}$.

With this relation, and the inferred time evolution of the jet Lorentz factor (Figure 3) we can infer the expected increase in energy. We find $\Gamma_{j} \approx 5.5$ at $\delta t=10 \mathrm{~d}$ and $\Gamma_{j} \approx 2.2$ at $\delta t=216$ $\mathrm{d}$, indicating an expected increase in energy by about a factor of 10 for our inferred profile. This is in excellent agreement with the data. Thus, a jet with a distribution of Lorentz factors of $E\left(>\Gamma_{j}\right) \propto \Gamma_{j}^{-2.5}$ naturally explains the evolution of source size and the substantial increase in energy beyond the on-going input from a $t^{-5 / 3}$ tail.

\subsection{The Radial Density Profile}

In addition to the substantial increase in energy, we also find that the normalization of the density profile, $n_{18} \equiv n_{\mathrm{CNM}} r_{18}^{2}$, changes as a function of time (Figure 3). This indicates that the radial density structure is not a simple Wind profile, for which $n_{18}$ is by definition constant. In particular, we find that $n_{18}$ decreases at $5-10 \mathrm{~d}$, followed by a steady increase at $10-216 \mathrm{~d}$ is about a factor of 8 . The resulting radial density profile is shown in Figure 6, and clearly reflects the complex evolution of $n_{18}$. The density profile at $r \approx 0.1-0.4 \mathrm{pc}$ is $\rho \propto$ $r^{-1.5}$, followed by a uniform density at $r \approx 0.4-0.6 \mathrm{pc}$, and a slow transition back to $\rho \propto r^{-1.5}$ by $r \approx 1.2 \mathrm{pc}$. The inferred density is $n \approx 0.2-2 \mathrm{~cm}^{-3}$ at $r \approx 1.2-0.1 \mathrm{pc}$, respectively. These values scale with the choice of equipartition fractions: for $\epsilon_{B}=0.01$ the density is about 3 times larger, while for $\epsilon_{e}=0.01$ it is about 1.7 times lower. Thus, the inferred density is fairly robust to large changes in the equipartition fractions.

The flattening at $r \approx 0.4-0.6 \mathrm{pc}$ coincides with the rapid increase in energy at $\delta t \approx 35-100 \mathrm{~d}$. This can be understood in the context of an outflow with a distribution of Lorentz factors since the relative increase in density compared to the previous $r^{-1.5}$ profile leads to enhanced contribution from lower Lorentz factor ejecta. A similar effect has been observed in the radio emission from some core-collapse supernovae, which have a steep ejecta profile with $E \propto v^{-5}$ (e.g., Chevalier 1982; Matzner \& McKee 1999; Berger et al. 2002; Soderberg et al. 2006).

Also shown in Figure 6are the inferred densities in the central parsec of the Milky way galactic center from X-ray measurements (Baganoff et al. 2003). The Galactic Center density is similar that inferred for Sw $1644+57$ at $r \approx 0.05 \mathrm{pc}$, but

\footnotetext{
0.01 .
} 
is about 30 times larger at $r \approx 0.4 \mathrm{pc}$. If the bulk of the gas in the central parsec is due to mass loss from massive stars (e.g., Melia 1992; Baganoff et al. 2003; Ouataert 2004), the lower density inferred here may indicate a much smaller number of massive stars in the central parsec of the host galaxy of Sw 1644+57. Although we cannot investigate star formation on scales smaller than $\sim 1 \mathrm{kpc}$ in the host of Sw 1644+57, the overall star formation rate in this galaxy is indeed a factor of several times lower than in the Milky Way (Levan et al. 2011). Regardless of the reason for the difference in density, it is quite remarkable that the radio emission from $\mathrm{Sw} 1644+57$ offers as detailed a view (or better) of the density profile in the inner parsec around an inactive SMBH at $z=0.354$ as available for the Galactic Center at a distance of only $8.5 \mathrm{kpc}$.

\subsection{Optical and Near-Infrared Emission}

Using the radio modeling we can also predict the optical and near-IR emission from the jet. In Figure 7 we plot the optical $r$-band upper limits from Levan et al. (2011), as well as their near-IR $K$-band measurements. The latter include the total flux from Sw 1644+57 and its host galaxy since transientfree templates are not presently available. We estimate the host contribution to be about $20 \mu \mathrm{Jy}(K \approx 20.6 \mathrm{AB} \mathrm{mag})$ by requiring that the overall shape of the $K$-band light curve match our predicted light curve. We note that this only affects the light curve shape at $\delta t \gtrsim 15 \mathrm{~d}$ since at earlier times the observed flux is dominated by the transient itself. A simple extrapolation of our model over-predicts the $K$-band flux density by about an order of magnitude, indicating the presence of an additional break in the spectrum between the radio and optical/near-IR band. Such a break is indicative of the synchrotron cooling frequency, $\nu_{c}$. To explain the $K$-band flux density requires $\nu_{c} \sim 10^{13} \mathrm{~Hz}$. Even if we include this break, the observed $r$-band limits are still a factor of about 70 times fainter than the model prediction. To explain this discrepancy requires host galaxy extinction of $A_{\mathrm{V} \text {,host }} \gtrsim 3.5 \mathrm{mag}$ (see also Levan et al. 2011, ZBS11). With the addition of a cooling break and host galaxy extinction we find an excellent match between our model and the near-IR fluxes. Since these data were not used in the fitting, the agreement between our model and the data provides an independent confirmation of the model.

\section{IMPLICATIONS FOR RELATIVISTIC JETS AND THE} ENVIRONMENTS OF SUPERMASSIVE BLACK HOLES

Our on-going radio observations have uncovered two unexpected and critical results regarding the nature of the relativistic outflow from Sw 1644+57 and the parsec-scale environment around a previously-dormant SMBH. We find that the relativistic outflow is not dominated by a single Lorentz factor, as typically assumed in GRB jet models. Instead, the outflow has a Lorentz factor distribution with $E_{j}\left(>\Gamma_{j}\right) \propto \Gamma_{j}^{-2.5}$, at least over the range of Lorentz factors probed so far, $\Gamma_{j} \approx$ $2.2-5.5$. This scenario is reminiscent of supernova ejecta, in which the energy is a strong function of velocity, $E \propto v^{-5}$ (Chevalier 1982; Matzner \& McKee 1999). In the SN case, this coupling reflects shock acceleration through the steep density gradient in the outer envelope of the star. In the case of Sw $1644+57$ we do not expect such a density gradient, suggesting instead that the ejecta structure may be an intrinsic property of relativistic jet launching, potentially through the Blandford-Znajek mechanism (Blandford \& Znajek 1977). In this case, the same structure may also apply to the relativistic jets of GRBs, which are generally assumed to have a single
Lorentz factor.

To assess this possibility we compare the ratio of event duration to the accretion disk dynamical timescale, $t_{\text {dyn }} \propto M_{\mathrm{BH}}\left(r / r_{g}\right)^{3 / 2}$, where $r_{g}=2 G M_{\mathrm{BH}} / c^{2}$ is the black hole Schwarzschild radius. For similar ratios of $\left(r / r_{g}\right)$, since $M_{\mathrm{BH}, \mathrm{Sw} 1644+57} \sim 10^{6} \mathrm{M}_{\odot} \sim 10^{5} M_{\mathrm{BH}, \mathrm{GRBs}}$, the dynamical timescale in $\mathrm{Sw} 1644+57$ is about $10^{5}$ times longer than in GRBs. The ratio of durations is similar, $\sim 10^{6} \mathrm{~s}$ for Sw $1644+57$ and $\sim 10$ s for GRBs. Therefore, the ratio of duration to dynamical timescale is similar, and the jet launching mechanism may imprint a similar profile in the case of Sw 1644+57 and GRBss. Of course, in long GRBs the jet still has to propagate through the stellar envelope, which may modify the jet structure.

We next address the potential implications of the radial density profile and the flattening at $r \approx 0.4-0.6 \mathrm{pc}$. One possibility is that this scale represents the Bondi radius for spherical accretion from the CNM, $r_{B}=G M / c_{s}^{2}=\left(c / c_{s}\right)^{2} r_{g} / 2$; here $c_{s}$ is the sound speed in the CNM and $r_{g}=2 G M / c^{2}$ is the Schwarzschild radius of the SMBH. For a CNM gas temperature of $\sim 10^{7} \mathrm{~K}$ (Baganoff et al. 2003; Narayan \& Fabian 2011), we find $r_{B} \approx 10^{6} r_{s} \approx 0.1-1 \mathrm{pc}$ for the SMBH mass of $10^{6}-10^{7} \mathrm{M}_{\odot}$ inferred for Sw $1644+57$. Thus, the radius at which we infer a density enhancement is in reasonable agreement with the expected Bondi radius. In addition, we note that the expected density profile inside the Bondi radius, $\rho \propto r^{-3 / 2}$, matches the inferred profile for Sw $1644+57$ on a scale of $\sim 0.15-0.4 \mathrm{pc}$. In the standard scenario, the medium outside the Bondi radius is assumed to have a uniform density, while here we infer a continued decline in the density at $r \approx 0.6-1.2 \mathrm{pc}$. This may reflect the complex conditions in the inner few parsecs around the SMBH in which the interstellar density profile may be mainly influenced by mass loss from stars (as in the case of the Milky Way galactic center; e.g., Krabbe et al. 1991). Indeed, from the inferred density of $n_{\mathrm{CNM}} \approx 0.6 \mathrm{~cm}^{-3}$ at $r \approx 0.5 \mathrm{pc}$, the required mass loss rate is $\dot{M} \approx 5 \times 10^{-5} \mathrm{M}_{\odot} \mathrm{yr}^{-1}$ for a wind velocity of $10^{3} \mathrm{~km} \mathrm{~s}^{-1}$.

In particular, in the case of the Galactic Center it has long been believed that the gas in the central parsec is supplied by mass loss from massive stars, with the bulk of the gas ( $\geq 90 \%$ ) being thermally expelled in a wind (Melia 1992; Baganoff et al. 2003; Ouataert 2004, but see Sazonov et al. 2011 for an alternative interpretation of the X-ray emission). Using a spherically symmetric hydrodynamic simulation of the gas supplied by stellar winds, Ouataert (2004) showed that beyond about $0.4 \mathrm{pc}$ gas is mainly expelled with a resulting Wind profile $\left(\rho \propto r^{-2}\right)$ on larger scales, while on scales of $\lesssim 0.2 \mathrm{pc}$ the profile is roughly as expected for Bondi accretion $\left(\rho \propto r^{-3 / 2}\right)$; see Figure 6 The profile we find here is somewhat different from this model, but this may be due to a different distribution of massive stars, or to the simplifying assumption of spherical symmetry in the model.

\section{CONCLUSIONS AND FUTURE DIRECTIONS}

We presented radio observations of Sw 1644+57 extending to $\delta t \approx 216 \mathrm{~d}$ and spanning a wide range of frequencies. The evolution of the radio emission changes dramatically at $\delta t \gtrsim 1$ month, requiring an increase in the total energy by about an order of magnitude, a density profile of $\rho \propto r^{-3 / 2}(0.1-1.2$ pc), and a flattening at $r \approx 0.4-0.6 \mathrm{pc}$. A comparison of the model to optical limits and near-IR detections indicates a cooling break at $\nu_{c} \sim 10^{13} \mathrm{~Hz}$ and host galaxy extinction of $A_{\mathrm{V}, \text { host }} \gtrsim 3.5$ mag. 
The increase in energy cannot be explained by injection from an $L \propto t^{-5 / 3}$ tail that is expected in tidal disruption events and which matches the evolution of the X-ray emission. We conclude that a natural explanation is a structured outflow with $E\left(>\Gamma_{j}\right) \propto \Gamma_{j}^{-2.5}$. The inferred density profile and the radial scale of the density enhancement are in rough agreement with the expectation for Bondi accretion from a circumnuclear medium. The jet energetics and structure, as well as the detailed density profile on $\sim 0.1-1 \mathrm{pc}$ scale are a testament to the important insight that can be gained from continued radio observations of Sw $1644+57$. In particular, the radial density profile is traced in greater detail than even the inner parsec of the Milky Way. Continued radio observations will probe the environment to a scale of $\sim 10 \mathrm{pc}$ in the coming decade.

Using the results of our analysis we can predict the future evolution of the radio emission (modulo any future unpredictable changes in energy and/or density as we have found here). We use the evolution of $L_{\mathrm{j} \text {,iso }}$ and $n_{\mathrm{CNM}}$ as inferred from the data at $\delta t \lesssim 216 \mathrm{~d}$, and assume that the density will continue to evolve as $\rho \propto r^{-1.5}$ and that the energy will increase to a maximum beaming-corrected value of $E_{j}=L_{\mathrm{j}, \text { iso }} t_{j}\left[1-\cos \left(\theta_{j}\right)\right]$ with $E_{j}=10^{52}, 3 \times 10^{52}, 10^{53} \mathrm{erg}$. The resulting light curves at 6 and $22 \mathrm{GHz}$ are shown in Figure 8 . The long-term evolution is marked by a break when $E_{j}$ achieves its maximum value, corresponding to about 5,28, and $180 \mathrm{yr}$ for our three choices of maximum energy. Using the $5 \sigma$ sensitivity of the EVLA in an observation of a few hours $^{14}$, we find that the emission at 22 and $6 \mathrm{GHz}$ should be detectable for at least $\sim 40 \mathrm{yr}$ and $\sim 80 \mathrm{yr}$, respectively. Indeed, any significant upgrades to the EVLA or the construction of more sensitive radio facilities in the coming decades may extend the range of detectability to centuries ${ }^{15}$. The same is true if the total energy scale is $\sim 10^{53} \mathrm{erg}$.

An equally important question is whether the jet will be resolvable with VLBI in the future. The projected radius is $r_{\text {proj }} \approx r \theta_{j}$, as long as the jet maintains its collimation. In Figure 9 we plot the predicted future evolution of $r$ using the prescription described above. We find that for $\theta_{j}=0.1$ and a best-case VLBI angular resolution of $\approx 0.2$ mas (FWHM), the source should become resolvable at $\delta t \approx 6$ yr. On this timescale the $22 \mathrm{GHz}$ flux density is expected to be only $\approx$ 2 mJy (Figure 8), still accessible with VLBI. While the flux density at $6 \mathrm{GHz}$ is expected to be larger by about a factor of 2.6, the angular resolution at this frequency is poorer by

\footnotetext{
${ }^{14}$ At $22 \mathrm{GHz}$ we use the sensitivity for the full $8 \mathrm{GHz}$ bandwidth that will become available some time in 2012 .

15 Significant budget cuts to radio facilities in the future may lead to the
}

about a factor of 3.7, making it less competitive than $22 \mathrm{GHz}$. Thus, we conclude that the radio emission from Sw 1644+57 may be marginally resolved in a few years. On the other hand, if the jet undergoes significant spreading on the timescale at which it becomes non-relativistic (as expected for GRB jets: e.g., Livio \& Waxman 2000) it is possible that it will become resolvable at $\delta t \sim 1-2 \mathrm{yr}$ when the expected $22 \mathrm{GHz}$ flux density is still $\sim 10 \mathrm{mJy}$.

We are undertaking continued multi-frequency radio monitoring of Sw 1644+57 to follow the long-term evolution of the relativistic outflow and the radial profile of the ambient medium. Even in the absence of any future dramatic changes relative to the current evolution, we expect that in the next few years we may be able to determine the total energy of the relativistic outflow, measure the spreading of the jet, and study the radial density profile to a scale of $\sim 10 \mathrm{pc}$. Future papers in this series will detail these results.

We thank Ramesh Narayan, Ryan Chornock, and Nicholas Stone for helpful discussions, and Glen Petitpas for assistance with the SMA data reduction. E.B. acknowledges support from Swift AO6 grant NNX10AI24G and from the National Science Foundation through Grant AST-1107973. A.B. is supported by a Marie Curie Outgoing International Fellowship (FP7) of the European Union (project number 275596). This work is partially based on observations with the 100$m$ telescope of the MPIfR (Max-Planck-Institut für Radioastronomie) at Effelsberg. The Submillimeter Array is a joint project between the Smithsonian Astrophysical Observatory and the Academia Sinica Institute of Astronomy and Astrophysics, and is funded by the Smithsonian Institution and the Academia Sinica. Support for CARMA construction was derived from the Gordon and Betty Moore Foundation, the Kenneth T. and Eileen L. Norris Foundation, the James S. McDonnell Foundation, the Associates of the California Institute of Technology, the University of Chicago, the states of California, Illinois, and Maryland, and the National Science Foundation. Ongoing CARMA development and operations are supported by the National Science Foundation under a cooperative agreement, and by the CARMA partner universities. The AMI arrays are supported by the University of Cambridge and the STFC. This work made use of data supplied by the UK Swift Science Data Centre at the University of Leicester.

\section{REFERENCES}

Baganoff, F. K., et al. 2003, ApJ, 591, 891

Beasley, A. J., Gordon, D., Peck, A. B., Petrov, L., MacMillan, D. S., Fomalont, E. B., \& Ma, C. 2002, ApJS, 141, 13

Berger, E., Kulkarni, S. R., \& Chevalier, R. A. 2002, ApJ, 577, L5

Bietenholz, M. F., et al. 2010, ApJ, 725, 4

Blandford, R. D., \& Znajek, R. L. 1977, MNRAS, 179, 433

Bloom, J. S., et al. 2011, Science, 333, 203

Brunthaler, A., Reid, M. J., \& Falcke, H. 2005, in Astronomical Society of the Pacific Conference Series, Vol. 340, Future Directions in High Resolution Astronomy, ed. J. Romney \& M. Reid, 455

Burrows, D. N., et al. 2011, Nature, 476, 421

Chevalier, R. A. 1982, ApJ, 258, 790

Gezari, S., et al. 2008, ApJ, 676, 944

Giannios, D., \& Metzger, B. D. 2011, MNRAS, 416, 2102

Granot, J., \& Sari, R. 2002, ApJ, 568, 820; GS02
Kettenis, M., van Langevelde, H. J., Reynolds, C., \& Cotton, B. 2006, in Astronomical Society of the Pacific Conference Series, Vol. 351, Astronomical Data Analysis Software and Systems XV, ed. C. Gabriel, C. Arviset, D. Ponz, \& S. Enrique, 497

Komossa, S., \& Greiner, J. 1999, A\&A, 349, L45

Krabbe, A., Genzel, R., Drapatz, S., \& Rotaciuc, V. 1991, ApJ, 382, L19

Krolik, J. H., \& Piran, T. 2011, ApJ, 743, 134

Levan, A. J., et al. 2011, Science, 333, 199

Livio, M., \& Waxman, E. 2000, ApJ, 538, 187

Ma, C., et al. 1998, AJ, 116, 516

Matzner, C. D., \& McKee, C. F. 1999, ApJ, 510, 379

Melia, F. 1992, ApJ, 387, L25

Metzger, B. D., Giannios, D., \& Mimica, P. 2011, arXiv:1110.1111 MGM11

Narayan, R., \& Fabian, A. C. 2011, MNRAS, 415, 3721

Ouyed, R., Staff, J. E., \& Jaikumar, P. 2011, ApJ, 743, 116

Quataert, E. 2004, ApJ, 613, 322

Quataert, E., \& Kasen, D. 2011, MNRAS, L360 
Rees, M. J. 1988, Nature, 333, 523

Reid, M. J., Menten, K. M., Brunthaler, A., Zheng, X. W., Moscadelli, L., \& Xu, Y. 2009, ApJ, 693, 397

Sari, R., \& Mészáros, P. 2000, ApJ, 535, L33

Sari, R., \& Piran, T. 1995, ApJ, 455, L143

Sazonov, S., Sunyaev, R., \& Revnivtsev, M. 2011, arXiv:1108.2778

Soderberg, A. M., Chevalier, R. A., Kulkarni, S. R., \& Frail, D. A. 2006, ApJ, 651,1005
Strubbe, L. E., \& Quataert, E. 2009, MNRAS, 400, 2070 van Velzen, S., et al. 2011, ApJ, 741, 73

Zauderer, B. A., et al. 2011, Nature, 476, 425; ZBS11 
TABLE 1

RADIO OBSERVATIONS OF SW 1644+57

\begin{tabular}{|c|c|c|c|}
\hline $\begin{array}{l}\delta t^{a} \\
\text { (d) }\end{array}$ & Facility & $\begin{array}{c}\text { Frequency } \\
\quad(\mathrm{GHz})\end{array}$ & $\begin{array}{c}\text { Flux Density } \\
(\mathrm{mJy})\end{array}$ \\
\hline 6.79 & EVLA & 1.4 & $0.21 \pm 0.08$ \\
\hline 126.59 & EVLA & 1.4 & $1.10 \pm 0.10$ \\
\hline 174.47 & EVLA & 1.4 & $1.60 \pm 0.11$ \\
\hline 197.41 & EVLA & 1.4 & $1.80 \pm 0.10$ \\
\hline 3.87 & $\overline{\text { EVLA }}$ & 4.9 & $0.25 \pm 0.02$ \\
\hline 4.76 & EVLA & 4.9 & $0.34 \pm 0.02$ \\
\hline 5.00 & EVLA & 4.9 & $0.34 \pm 0.02$ \\
\hline 5.79 & EVLA & 4.9 & $0.61 \pm 0.02$ \\
\hline 6.78 & EVLA & 4.9 & $0.82 \pm 0.02$ \\
\hline 7.77 & EVLA & 4.9 & $1.48 \pm 0.02$ \\
\hline 9.79 & EVLA & 4.9 & $1.47 \pm 0.02$ \\
\hline 14.98 & EVLA & 4.9 & $1.80 \pm 0.03$ \\
\hline 22.78 & EVLA & 4.9 & $2.10 \pm 0.01$ \\
\hline 35.86 & EVLA & 4.9 & $4.62 \pm 0.02$ \\
\hline 50.65 & EVLA & 4.9 & $4.84 \pm 0.03$ \\
\hline 67.61 & EVLA & 4.9 & $5.86 \pm 0.03$ \\
\hline 94.64 & EVLA & 4.9 & $9.06 \pm 0.03$ \\
\hline 111.62 & EVLA & 4.9 & $9.10 \pm 0.03$ \\
\hline 126.51 & EVLA & 4.9 & $9.10 \pm 0.03$ \\
\hline 143.62 & EVLA & 4.9 & $11.71 \pm 0.03$ \\
\hline 164.38 & EVLA & 4.9 & $12.93 \pm 0.05$ \\
\hline 174.47 & EVLA & 4.9 & $12.83 \pm 0.06$ \\
\hline 197.41 & EVLA & 4.9 & $13.29 \pm 0.03$ \\
\hline 213.32 & EVLA & 4.9 & $12.43 \pm 0.04$ \\
\hline $\begin{array}{l}3.87 \\
\end{array}$ & 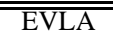 & 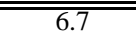 & $=0.38 \pm 0.02$ \\
\hline 4.76 & EVLA & 6.7 & $0.63 \pm 0.02$ \\
\hline 5.00 & EVLA & 6.7 & $0.64 \pm 0.02$ \\
\hline 5.79 & EVLA & 6.7 & $1.16 \pm 0.02$ \\
\hline 6.79 & EVLA & 6.7 & $1.47 \pm 0.02$ \\
\hline 7.77 & EVLA & 6.7 & $1.50 \pm 0.02$ \\
\hline 9.79 & EVLA & 6.7 & $2.15 \pm 0.02$ \\
\hline 14.98 & EVLA & 6.7 & $3.79 \pm 0.03$ \\
\hline 22.78 & EVLA & 6.7 & $3.44 \pm 0.01$ \\
\hline 35.86 & EVLA & 6.7 & $6.39 \pm 0.02$ \\
\hline 50.65 & EVLA & 6.7 & $5.70 \pm 0.02$ \\
\hline 67.61 & EVLA & 6.7 & $8.94 \pm 0.03$ \\
\hline 94.64 & EVLA & 6.7 & $13.43 \pm 0.03$ \\
\hline 111.62 & EVLA & 6.7 & $13.66 \pm 0.03$ \\
\hline 126.51 & EVLA & 6.7 & $14.16 \pm 0.04$ \\
\hline 143.62 & EVLA & 6.7 & $16.85 \pm 0.04$ \\
\hline 164.38 & EVLA & 6.7 & $18.27 \pm 0.06$ \\
\hline 174.47 & EVLA & 6.7 & $19.59 \pm 0.16$ \\
\hline 197.41 & EVLA & 6.7 & $19.34 \pm 0.03$ \\
\hline 213.32 & EVLA & 6.7 & $18.02 \pm 0.05$ \\
\hline $\begin{array}{c}14.97 \\
\end{array}$ & 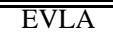 & 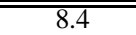 & $\overline{c 5.49 \pm 0.09}$ \\
\hline 127.69 & EVLA & 8.4 & $19.03 \pm 0.14$ \\
\hline 159.77 & EVLA & 8.4 & $22.15 \pm 0.20$ \\
\hline 174.47 & EVLA & 8.4 & $23.19 \pm 0.38$ \\
\hline 177.50 & EVLA & 8.4 & $23.65 \pm 0.16$ \\
\hline 197.41 & EVLA & 8.4 & $22.42 \pm 0.10$ \\
\hline 213.32 & EVLA & 8.4 & $22.04 \pm 0.13$ \\
\hline 219.22 & EVLA & 8.4 & $21.52 \pm 0.09$ \\
\hline $\begin{array}{l}5.81 \\
\end{array}$ & 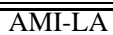 & $\overline{15.4}$ & $=2.69 \pm 0.44$ \\
\hline 6.64 & AMI-LA & 15.4 & $3.62 \pm 0.22$ \\
\hline 7.62 & AMI-LA & 15.4 & $4.32 \pm 0.28$ \\
\hline 8.55 & AMI-LA & 15.4 & $5.07 \pm 0.36$ \\
\hline 9.56 & AMI-LA & 15.4 & $6.68 \pm 0.51$ \\
\hline 10.78 & AMI-LA & 15.4 & $6.74 \pm 0.48$ \\
\hline 11.56 & AMI-LA & 15.4 & $7.50 \pm 0.44$ \\
\hline 13.64 & AMI-LA & 15.4 & $8.02 \pm 0.71$ \\
\hline 14.57 & AMI-LA & 15.4 & $8.43 \pm 0.32$ \\
\hline 16.47 & AMI-LA & 15.4 & $8.86 \pm 0.58$ \\
\hline 18.65 & AMI-LA & 15.4 & $8.62 \pm 0.40$ \\
\hline 19.73 & AMI-LA & 15.4 & $10.04 \pm 0.63$ \\
\hline 21.78 & AMI-LA & 15.4 & $10.91 \pm 0.89$ \\
\hline 22.76 & AMI-LA & 15.4 & $11.01 \pm 0.77$ \\
\hline 25.38 & AMI-LA & 15.4 & $10.28 \pm 0.58$ \\
\hline 26.74 & AMI-LA & 15.4 & $11.36 \pm 0.80$ \\
\hline 31.45 & AMI-LA & 15.4 & $11.24 \pm 0.41$ \\
\hline 33.61 & AMI-LA & 15.4 & $12.14 \pm 0.16$ \\
\hline 34.79 & AMI-LA & 15.4 & $11.89 \pm 0.25$ \\
\hline 35.71 & AMI-LA & 15.4 & $13.39 \pm 0.49$ \\
\hline 37.49 & AMI-LA & 15.4 & $13.63 \pm 0.45$ \\
\hline 38.60 & AMI-LA & 15.4 & $13.72 \pm 0.39$ \\
\hline
\end{tabular}


TABLE 1 - Continued

\begin{tabular}{|c|c|c|c|}
\hline $\begin{array}{l}\delta t^{a} \\
\text { (d) }\end{array}$ & Facility & $\begin{array}{l}\text { Frequency } \\
(\mathrm{GHz})\end{array}$ & $\begin{array}{c}\text { Flux Density } \\
\text { (mJy) }\end{array}$ \\
\hline 39.78 & AMI-LA & 15.4 & $10.80 \pm 0.91$ \\
\hline 40.67 & AMI-LA & 15.4 & $13.38 \pm 0.40$ \\
\hline 41.69 & AMI-LA & 15.4 & $13.64 \pm 0.21$ \\
\hline 43.63 & AMI-LA & 15.4 & $14.21 \pm 1.24$ \\
\hline 45.74 & AMI-LA & 15.4 & $12.33 \pm 0.37$ \\
\hline 48.69 & AMI-LA & 15.4 & $14.06 \pm 0.41$ \\
\hline 49.64 & AMI-LA & 15.4 & $13.94 \pm 0.05$ \\
\hline 50.54 & AMI-LA & 15.4 & $14.39 \pm 0.25$ \\
\hline 53.39 & AMI-LA & 15.4 & $15.94 \pm 0.59$ \\
\hline 55.62 & AMI-LA & 15.4 & $15.28 \pm 0.33$ \\
\hline 56.52 & AMI-LA & 15.4 & $17.91 \pm 0.07$ \\
\hline 60.73 & AMI-LA & 15.4 & $15.00 \pm 1.18$ \\
\hline 62.54 & AMI-LA & 15.4 & $19.23 \pm 0.62$ \\
\hline 63.45 & AMI-LA & 15.4 & $16.47 \pm 0.21$ \\
\hline 65.51 & AMI-LA & 15.4 & $18.77 \pm 0.37$ \\
\hline 68.60 & AMI-LA & 15.4 & $19.06 \pm 0.48$ \\
\hline 70.69 & AMI-LA & 15.4 & $20.34 \pm 0.38$ \\
\hline 71.38 & AMI-LA & 15.4 & $19.36 \pm 0.56$ \\
\hline 73.48 & AMI-LA & 15.4 & $21.27 \pm 0.34$ \\
\hline 74.48 & AMI-LA & 15.4 & $21.84 \pm 0.59$ \\
\hline 76.65 & AMI-LA & 15.4 & $21.40 \pm 0.51$ \\
\hline 77.47 & AMI-LA & 15.4 & $23.08 \pm 0.26$ \\
\hline 78.49 & AMI-LA & 15.4 & $23.26 \pm 0.65$ \\
\hline 79.49 & AMI-LA & 15.4 & $23.22 \pm 0.24$ \\
\hline 80.47 & AMI-LA & 15.4 & $22.94 \pm 0.50$ \\
\hline 81.48 & AMI-LA & 15.4 & $21.74 \pm 0.45$ \\
\hline 83.42 & AMI-LA & 15.4 & $23.99 \pm 0.50$ \\
\hline 86.65 & AMI-LA & 15.4 & $22.94 \pm 0.33$ \\
\hline 88.37 & AMI-LA & 15.4 & $24.69 \pm 0.26$ \\
\hline 89.64 & AMI-LA & 15.4 & $25.90 \pm 0.50$ \\
\hline 91.58 & AMI-LA & 15.4 & $26.07 \pm 1.04$ \\
\hline 92.58 & AMI-LA & 15.4 & $25.40 \pm 0.55$ \\
\hline 95.39 & AMI-LA & 15.4 & $25.34 \pm 0.72$ \\
\hline 98.42 & AMI-LA & 15.4 & $26.15 \pm 0.25$ \\
\hline 100.61 & AMI-LA & 15.4 & $27.83 \pm 0.57$ \\
\hline 101.60 & AMI-LA & 15.4 & $25.96 \pm 0.91$ \\
\hline 102.60 & AMI-LA & 15.4 & $26.60 \pm 0.26$ \\
\hline 105.59 & AMI-LA & 15.4 & $26.68 \pm 0.21$ \\
\hline 107.38 & AMI-LA & 15.4 & $27.89 \pm 0.33$ \\
\hline 108.32 & AMI-LA & 15.4 & $26.84 \pm 0.40$ \\
\hline 110.34 & AMI-LA & 15.4 & $28.24 \pm 0.22$ \\
\hline 112.50 & AMI-LA & 15.4 & $27.10 \pm 0.58$ \\
\hline 113.50 & AMI-LA & 15.4 & $28.82 \pm 0.31$ \\
\hline 115.49 & AMI-LA & 15.4 & $28.39 \pm 0.23$ \\
\hline 118.31 & AMI-LA & 15.4 & $28.58 \pm 0.47$ \\
\hline 119.48 & AMI-LA & 15.4 & $26.90 \pm 0.80$ \\
\hline 120.55 & AMI-LA & 15.4 & $27.88 \pm 0.35$ \\
\hline 121.55 & AMI-LA & 15.4 & $26.92 \pm 0.73$ \\
\hline 122.18 & AMI-LA & 15.4 & $29.87 \pm 0.61$ \\
\hline 123.45 & AMI-LA & 15.4 & $28.86 \pm 0.41$ \\
\hline 124.38 & AMI-LA & 15.4 & $27.46 \pm 0.97$ \\
\hline 125.51 & AMI-LA & 15.4 & $27.40 \pm 0.56$ \\
\hline 127.38 & AMI-LA & 15.4 & $28.96 \pm 0.39$ \\
\hline 128.35 & AMI-LA & 15.4 & $28.69 \pm 1.00$ \\
\hline 130.38 & AMI-LA & 15.4 & $27.87 \pm 0.64$ \\
\hline 131.41 & AMI-LA & 15.4 & $28.94 \pm 1.11$ \\
\hline 132.49 & AMI-LA & 15.4 & $29.39 \pm 0.96$ \\
\hline 133.35 & AMI-LA & 15.4 & $30.81 \pm 1.03$ \\
\hline 134.36 & AMI-LA & 15.4 & $29.73 \pm 0.52$ \\
\hline 135.32 & AMI-LA & 15.4 & $31.25 \pm 0.59$ \\
\hline 136.46 & AMI-LA & 15.4 & $29.31 \pm 1.29$ \\
\hline 137.51 & AMI-LA & 15.4 & $29.58 \pm 0.23$ \\
\hline 138.51 & AMI-LA & 15.4 & $28.50 \pm 0.69$ \\
\hline 139.51 & AMI-LA & 15.4 & $28.96 \pm 0.57$ \\
\hline 140.50 & AMI-LA & 15.4 & $29.22 \pm 0.52$ \\
\hline 141.13 & AMI-LA & 15.4 & $29.03 \pm 0.45$ \\
\hline 142.30 & AMI-LA & 15.4 & $26.60 \pm 0.31$ \\
\hline 143.26 & AMI-LA & 15.4 & $28.96 \pm 0.61$ \\
\hline 144.42 & AMI-LA & 15.4 & $28.36 \pm 0.57$ \\
\hline 146.48 & AMI-LA & 15.4 & $28.92 \pm 0.64$ \\
\hline 147.48 & AMI-LA & 15.4 & $27.44 \pm 0.76$ \\
\hline 148.27 & AMI-LA & 15.4 & $29.49 \pm 0.97$ \\
\hline 149.27 & AMI-LA & 15.4 & $29.82 \pm 0.43$ \\
\hline 150.47 & AMI-LA & 15.4 & $29.83 \pm 0.23$ \\
\hline 151.22 & AMI-LA & 15.4 & $29.31 \pm 0.73$ \\
\hline 152.27 & AMI-LA & 15.4 & $28.10 \pm 1.30$ \\
\hline
\end{tabular}


TABLE 1 - Continued

\begin{tabular}{|c|c|c|c|}
\hline $\begin{array}{l}\delta t^{a} \\
(\mathrm{~d})\end{array}$ & Facility & $\begin{array}{c}\text { Frequency } \\
(\mathrm{GHz})\end{array}$ & $\begin{array}{c}\text { Flux Density } \\
\text { (mJy) }\end{array}$ \\
\hline 153.46 & AMI-LA & 15.4 & $26.71 \pm 0.38$ \\
\hline 155.45 & AMI-LA & 15.4 & $27.80 \pm 0.45$ \\
\hline 156.35 & AMI-LA & 15.4 & $29.92 \pm 0.89$ \\
\hline 157.45 & AMI-LA & 15.4 & $27.77 \pm 0.31$ \\
\hline 158.29 & AMI-LA & 15.4 & $27.72 \pm 0.83$ \\
\hline 159.28 & AMI-LA & 15.4 & $27.98 \pm 0.59$ \\
\hline 160.27 & AMI-LA & 15.4 & $28.93 \pm 0.44$ \\
\hline 161.20 & AMI-LA & 15.4 & $26.94 \pm 0.84$ \\
\hline 162.21 & AMI-LA & 15.4 & $26.81 \pm 1.21$ \\
\hline 163.28 & AMI-LA & 15.4 & $28.82 \pm 0.68$ \\
\hline 166.00 & AMI-LA & 15.4 & $26.92 \pm 0.58$ \\
\hline 168.13 & AMI-LA & 15.4 & $25.90 \pm 0.64$ \\
\hline 169.13 & AMI-LA & 15.4 & $25.47 \pm 0.83$ \\
\hline 172.25 & AMI-LA & 15.4 & $26.12 \pm 0.48$ \\
\hline 174.33 & AMI-LA & 15.4 & $26.12 \pm 0.15$ \\
\hline 177.32 & AMI-LA & 15.4 & $26.31 \pm 0.37$ \\
\hline 181.30 & AMI-LA & 15.4 & $24.37 \pm 0.85$ \\
\hline 184.03 & AMI-LA & 15.4 & $23.06 \pm 0.77$ \\
\hline 187.19 & AMI-LA & 15.4 & $24.24 \pm 0.43$ \\
\hline 187.91 & AMI-LA & 15.4 & $24.28 \pm 0.45$ \\
\hline 189.37 & AMI-LA & 15.4 & $24.60 \pm 0.59$ \\
\hline 193.19 & AMI-LA & 15.4 & $26.15 \pm 0.75$ \\
\hline 194.01 & AMI-LA & 15.4 & $24.53 \pm 0.34$ \\
\hline 197.02 & AMI-LA & 15.4 & $26.72 \pm 0.52$ \\
\hline 200.01 & AMI-LA & 15.4 & $25.90 \pm 0.15$ \\
\hline 203.01 & AMI-LA & 15.4 & $24.12 \pm 0.41$ \\
\hline 207.08 & AMI-LA & 15.4 & $25.06 \pm 0.45$ \\
\hline 210.19 & AMI-LA & 15.4 & $24.24 \pm 0.31$ \\
\hline 214.17 & AMI-LA & 15.4 & $22.64 \pm 0.38$ \\
\hline 217.16 & AMI-LA & 15.4 & $24.16 \pm 0.25$ \\
\hline 220.20 & AMI-LA & 15.4 & $23.74 \pm 0.05$ \\
\hline 221.22 & AMI-LA & 15.4 & $24.33 \pm 0.49$ \\
\hline 225.91 & AMI-LA & 15.4 & $23.80 \pm 0.14$ \\
\hline 232.87 & AMI-LA & 15.4 & $23.72 \pm 0.16$ \\
\hline 235.01 & AMI-LA & 15.4 & $21.64 \pm 0.16$ \\
\hline 237.85 & AMI-LA & 15.4 & $20.86 \pm 0.75$ \\
\hline 4.79 & EVLA & 19.1 & $2.12 \pm 0.02$ \\
\hline 6.75 & EVLA & 19.1 & $4.36 \pm 0.05$ \\
\hline 7.77 & EVLA & 19.1 & $5.25 \pm 0.03$ \\
\hline 8.87 & EVLA & 19.1 & $6.38 \pm 0.06$ \\
\hline 9.78 & EVLA & 19.1 & $5.42 \pm 0.03$ \\
\hline 21.89 & EVLA & 19.1 & $12.01 \pm 0.03$ \\
\hline 31.74 & EVLA & 19.1 & $13.50 \pm 0.05$ \\
\hline 35.86 & EVLA & 19.1 & $13.97 \pm 0.05$ \\
\hline 50.65 & EVLA & 19.1 & $17.11 \pm 0.06$ \\
\hline 67.61 & EVLA & 19.1 & $23.03 \pm 0.06$ \\
\hline 94.64 & EVLA & 19.1 & $31.36 \pm 0.07$ \\
\hline 111.62 & EVLA & 19.1 & $30.21 \pm 0.10$ \\
\hline 127.83 & EVLA & 19.1 & $29.75 \pm 0.22$ \\
\hline 142.62 & EVLA & 19.1 & $29.57 \pm 0.13$ \\
\hline 159.77 & EVLA & 19.1 & $26.10 \pm 0.26$ \\
\hline 177.50 & EVLA & 19.1 & $24.24 \pm 0.16$ \\
\hline 198.22 & EVLA & 19.1 & $23.02 \pm 0.12$ \\
\hline 219.22 & EVLA & 19.1 & $23.15 \pm 0.07$ \\
\hline 4.79 & EVLA & 24.4 & $3.01 \pm 0.03$ \\
\hline 6.75 & EVLA & 24.4 & $5.58 \pm 0.06$ \\
\hline 7.77 & EVLA & 24.4 & $6.70 \pm 0.03$ \\
\hline 8.87 & EVLA & 24.4 & $7.88 \pm 0.12$ \\
\hline 9.78 & EVLA & 24.4 & $6.84 \pm 0.03$ \\
\hline 21.89 & EVLA & 24.4 & $12.69 \pm 0.02$ \\
\hline 31.74 & EVLA & 24.4 & $13.80 \pm 0.06$ \\
\hline 35.86 & EVLA & 24.4 & $14.95 \pm 0.05$ \\
\hline 50.65 & EVLA & 24.4 & $18.30 \pm 0.06$ \\
\hline 67.61 & EVLA & 24.4 & $25.62 \pm 0.06$ \\
\hline 94.64 & EVLA & 24.4 & $30.67 \pm 0.07$ \\
\hline 111.62 & EVLA & 24.4 & $28.20 \pm 0.13$ \\
\hline 127.83 & EVLA & 24.4 & $28.29 \pm 0.33$ \\
\hline 142.62 & EVLA & 24.4 & $24.73 \pm 0.16$ \\
\hline 159.77 & EVLA & 24.4 & $23.83 \pm 0.40$ \\
\hline 177.50 & EVLA & 24.4 & $20.40 \pm 0.19$ \\
\hline 198.22 & EVLA & 24.4 & $19.88 \pm 0.15$ \\
\hline 219.22 & EVLA & 24.4 & $21.40 \pm 0.08$ \\
\hline 5.75 & $\overline{\text { EVLA }}$ & 43.6 & $8.11 \pm 0.16$ \\
\hline 6.75 & EVLA & 43.6 & $7.70 \pm 0.14$ \\
\hline 8.87 & EVLA & 43.6 & $9.62 \pm 0.14$ \\
\hline
\end{tabular}


TABLE 1 - Continued

\begin{tabular}{rccc}
\hline \hline $\begin{array}{c}\delta t^{a} \\
(\mathrm{~d})\end{array}$ & Facility & $\begin{array}{c}\text { Frequency } \\
(\mathrm{GHz})\end{array}$ & $\begin{array}{c}\text { Flux Density } \\
(\mathrm{mJy})\end{array}$ \\
\hline 111.62 & EVLA & 43.6 & $22.09 \pm 0.65$ \\
127.83 & EVLA & 43.6 & $20.84 \pm 0.48$ \\
159.77 & EVLA & 43.6 & $15.43 \pm 0.63$ \\
177.50 & EVLA & 43.6 & $15.73 \pm 0.38$ \\
198.22 & EVLA & 43.6 & $11.70 \pm 0.26$ \\
219.22 & EVLA & 43.6 & $15.32 \pm 0.15$ \\
\hline \hline 4.90 & CARMA & 87 & $15.66 \pm 0.51$ \\
8.19 & CARMA & 87 & $20.16 \pm 0.96$ \\
9.14 & CARMA & 87 & $21.67 \pm 0.33$ \\
10.23 & CARMA & 87 & $14.70 \pm 1.28$ \\
12.14 & CARMA & 87 & $17.67 \pm 0.94$ \\
17.66 & CARMA & 87 & $12.15 \pm 1.32$ \\
22.11 & CARMA & 87 & $11.70 \pm 1.74$ \\
25.12 & CARMA & 87 & $16.95 \pm 3.48$ \\
99.75 & CARMA & 87 & $18.79 \pm 0.65$ \\
131.52 & CARMA & 87 & $15.18 \pm 0.35$ \\
148.66 & CARMA & 87 & $11.88 \pm 0.35$ \\
175.56 & CARMA & 87 & $9.39 \pm 0.31$ \\
\hline \hline 19.25 & SMA & 200 & $14.10 \pm 1.50$ \\
24.32 & SMA & 200 & $10.70 \pm 1.00$ \\
\hline \hline 10.30 & SMA & 230 & $14.90 \pm 1.50$ \\
11.13 & SMA & 230 & $11.70 \pm 1.40$ \\
17.23 & SMA & 230 & $13.30 \pm 1.50$ \\
18.25 & SMA & 230 & $9.90 \pm 1.40$ \\
20.24 & SMA & 230 & $8.20 \pm 1.40$ \\
21.25 & SMA & 230 & $8.30 \pm 2.20$ \\
125.05 & SMA & 230 & $6.10 \pm 0.65$ \\
\hline \hline 5.13 & SMA & 345 & $35.10 \pm 0.80$ \\
\hline & & & \\
\hline
\end{tabular}

NOTE. $-{ }^{a}$ All values of $\delta t$ are relative to the initial $\gamma$-ray detection: 2011 March 25.5 UT. 
TABLE 2

RESUlts of BROAD-BAND SPECTRAL ENERgy Distribution Fits

\begin{tabular}{|c|c|c|c|c|c|c|c|c|c|c|}
\hline $\begin{array}{l}\delta t \\
\text { (d) }\end{array}$ & $\begin{array}{c}\log \left(\nu_{a}\right) \\
(\mathrm{Hz})\end{array}$ & $\begin{array}{c}\log \left(\nu_{m}\right) \\
(\mathrm{Hz})\end{array}$ & $\begin{array}{c}\log \left(F_{\nu_{a}}\right) \\
(\mathrm{mJy})\end{array}$ & $\begin{array}{c}\log \left(r_{18}\right) \\
(\mathrm{cm})\end{array}$ & $\log \left(\Gamma_{\mathrm{sh}}\right)$ & $\log \left(\Gamma_{j}\right)$ & $\begin{array}{c}\log \left(L_{\mathrm{j}, \text { iso }}, 4\right) \\
\left(\mathrm{erg} \mathrm{s}^{-1}\right)\end{array}$ & $\begin{array}{c}\log \left(n_{18}\right) \\
\left(\mathrm{cm}^{-3}\right)\end{array}$ & $\begin{array}{c}\log \left(n_{\mathrm{CNM}}\right) \\
\left(\mathrm{cm}^{-3}\right)\end{array}$ & $\begin{array}{l}\log \left(n_{j}\right) \\
\left(\mathrm{cm}^{-3}\right)\end{array}$ \\
\hline 5 & 11.01 & 11.74 & 1.47 & -0.79 & 0.65 & 0.78 & -1.33 & 0.28 & 1.82 & 3.53 \\
\hline 10 & 10.11 & 11.41 & 0.96 & -0.26 & 0.61 & 0.74 & -1.39 & -0.28 & 0.25 & 2.39 \\
\hline 15 & 10.02 & 11.21 & 0.98 & -0.13 & 0.55 & 0.65 & -1.33 & -0.22 & 0.05 & 2.19 \\
\hline 22 & 9.96 & 10.99 & 0.97 & -0.04 & 0.51 & 0.60 & -1.25 & -0.15 & -0.07 & 2.08 \\
\hline 36 & 9.95 & 10.78 & 1.11 & 0.08 & 0.47 & 0.54 & -1.05 & 0.01 & -0.17 & 2.03 \\
\hline 51 & 9.96 & 10.62 & 1.21 & 0.16 & 0.43 & 0.50 & -0.92 & 0.13 & -0.18 & 2.01 \\
\hline 68 & 9.99 & 10.53 & 1.39 & 0.24 & 0.41 & 0.47 & -0.72 & 0.28 & -0.20 & 2.04 \\
\hline 97 & 9.95 & 10.39 & 1.52 & 0.36 & 0.40 & 0.45 & -0.53 & 0.39 & -0.34 & 1.99 \\
\hline 126 & 9.97 & 10.26 & 1.58 & 0.41 & 0.36 & 0.41 & -0.46 & 0.49 & -0.33 & 1.97 \\
\hline 161 & 9.82 & 10.13 & 1.51 & 0.52 & 0.36 & 0.40 & -0.39 & 0.44 & -0.60 & 1.82 \\
\hline 197 & 9.83 & 10.04 & 1.56 & 0.56 & 0.34 & 0.38 & -0.32 & 0.52 & -0.60 & 1.81 \\
\hline 216 & 9.90 & 9.99 & 1.63 & 0.55 & 0.32 & 0.35 & -0.29 & 0.60 & -0.50 & 1.85 \\
\hline
\end{tabular}

NOTE. - Inferred parameters of the relativistic outflow and environment of Sw 1644+57 from model fits of individual multi-frequency epochs. The model is described in $\$ 4$ 


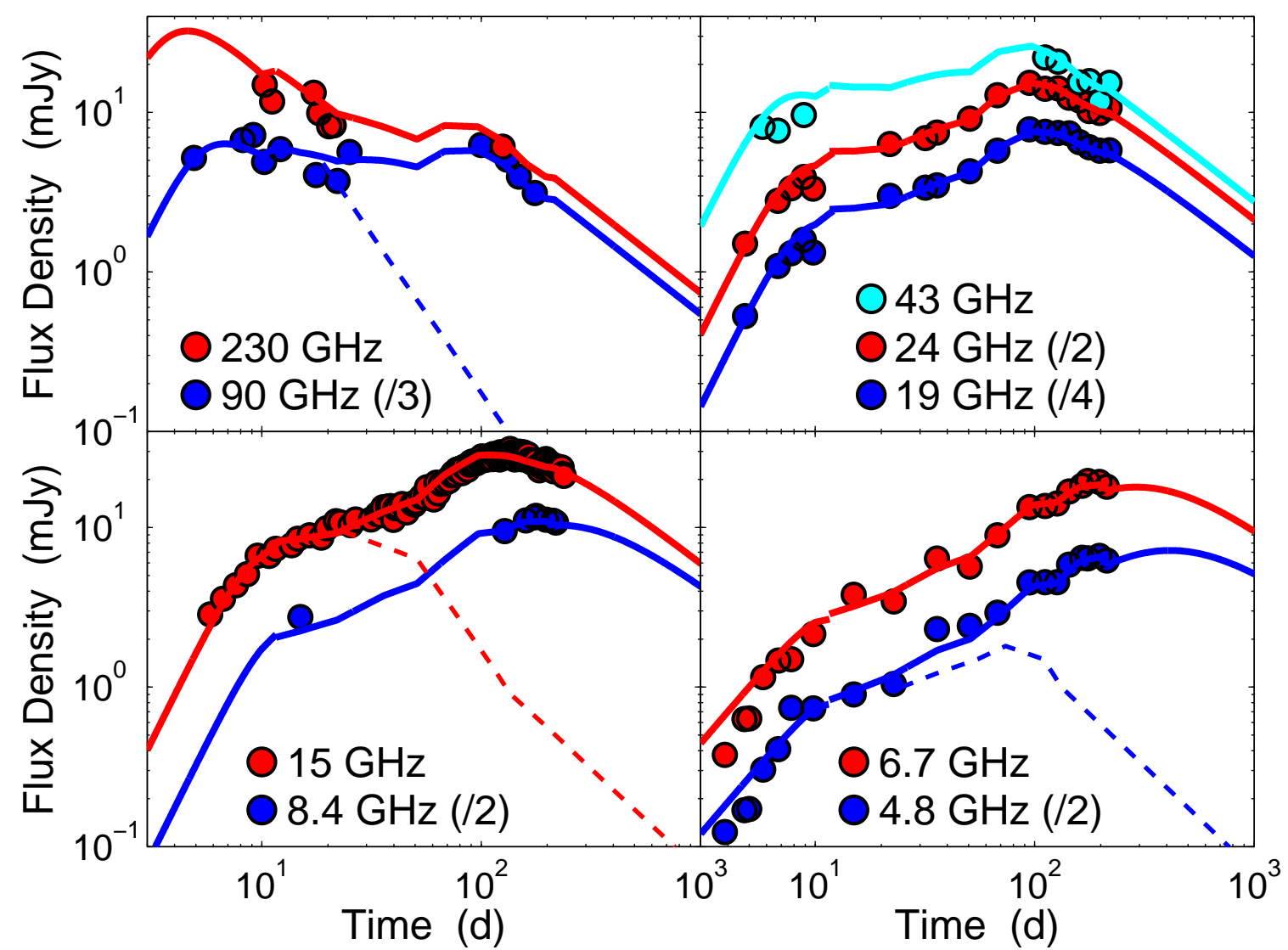

FIG. 1.- Radio light curves of Sw $1644+57$ extending to $\delta t \approx 216 \mathrm{~d}$. The data at $\delta t \approx 5-22 \mathrm{~d}$ were previously presented in ZBS11. The solid lines are models based on independent fits of broad-band SEDs (Figure 2) using the model described in $\$$ (see also MGM11). The dashed lines are the predicted light curves from MGM11, which assumed a constant energy and a steady density profile of $\rho \propto r^{-2}$. The secondary maximum in the millimeter band and the continued increase in brightness to a peak time of $\delta t \gtrsim 100-200 \mathrm{~d}$ in the centimeter bands require an increase in the energy and density relative to the initial evolution. 


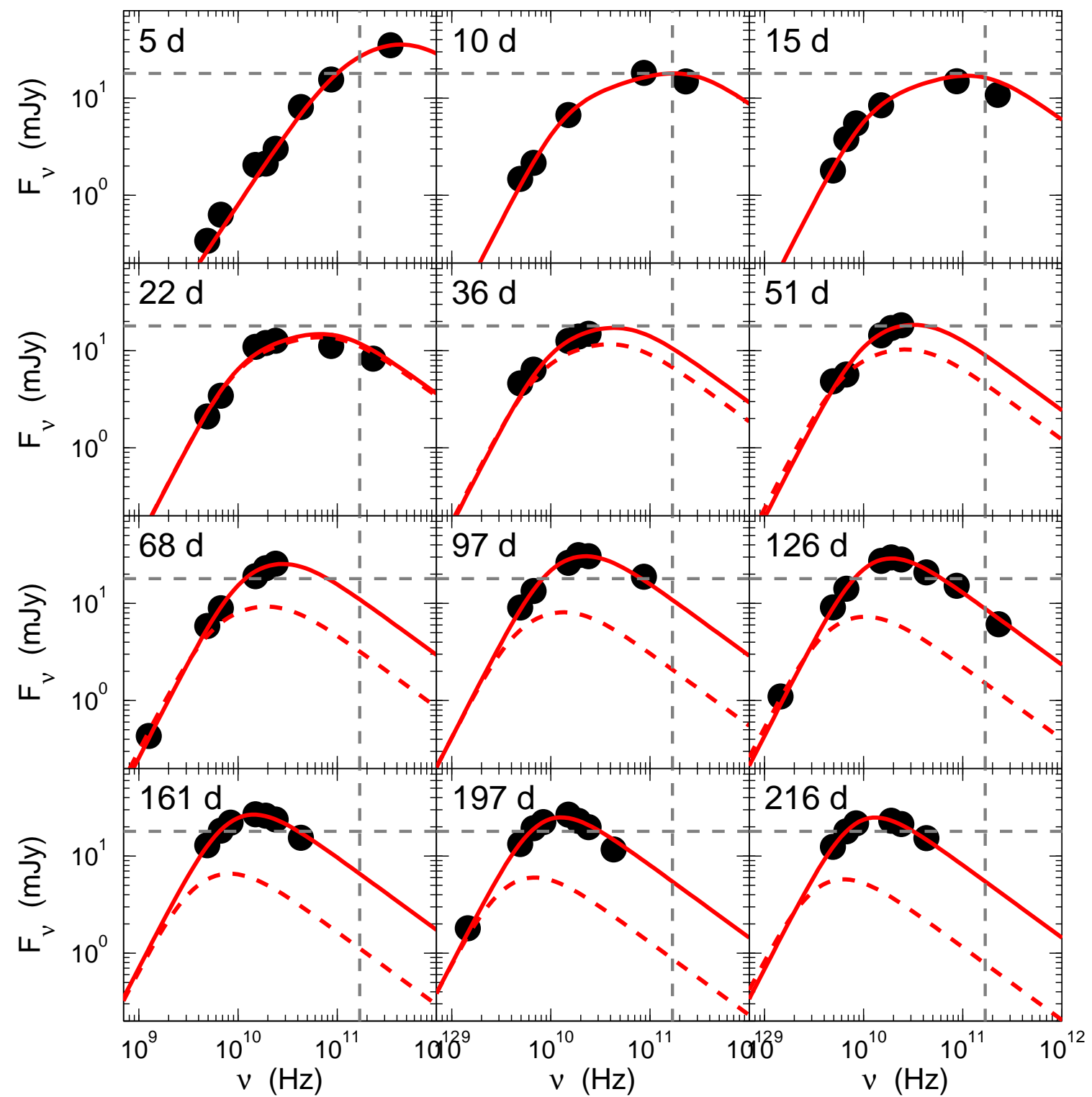

FIG. 2.- Radio spectral energy distributions of Sw $1644+57$ at $\delta t \approx 5-216 \mathrm{~d}$. The data at $\delta t \approx 5-22 \mathrm{~d}$ were previously presented in ZBS11. The solid lines

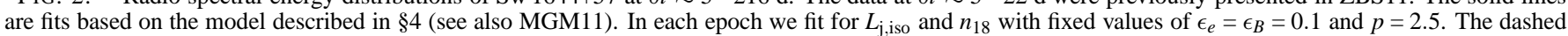
gray lines mark the peak flux density and peak frequency at $\delta t=10 \mathrm{~d}$ to help track the evolution of the spectrum as a function of time. The dashed red lines mark the expected SEDs based on the evolution at $\delta t \approx 5-22 \mathrm{~d}$. 

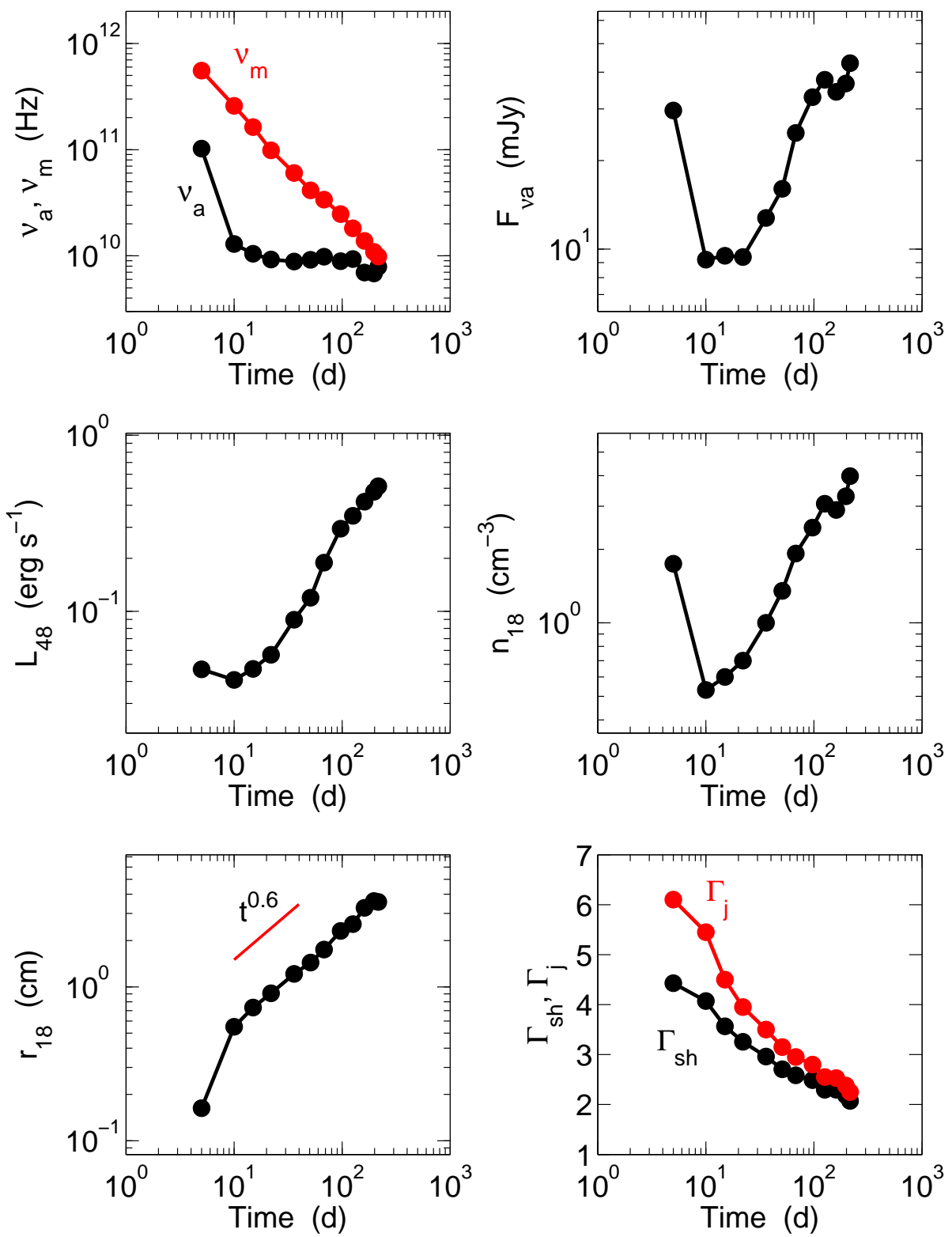

FIG. 3.- Model and extracted parameters for each broad-band SED shown in Figure 2 Shown are the time evolution of the synchrotron parameters $\left(\nu_{a}, \nu_{m}\right.$, and $\left.F_{\nu_{a}}\right), L_{\mathrm{j}, \text { iso,48, }}, n_{18}, r, \Gamma_{\mathrm{sh}}$, and $\Gamma_{j}$. The substantial increase in energy and density is clearly seen. In addition, we find $r \propto t^{0.6}$, a steeper increase than expected in a simple Wind model with constant energy $\left(r \propto t^{0.5}\right)$. 


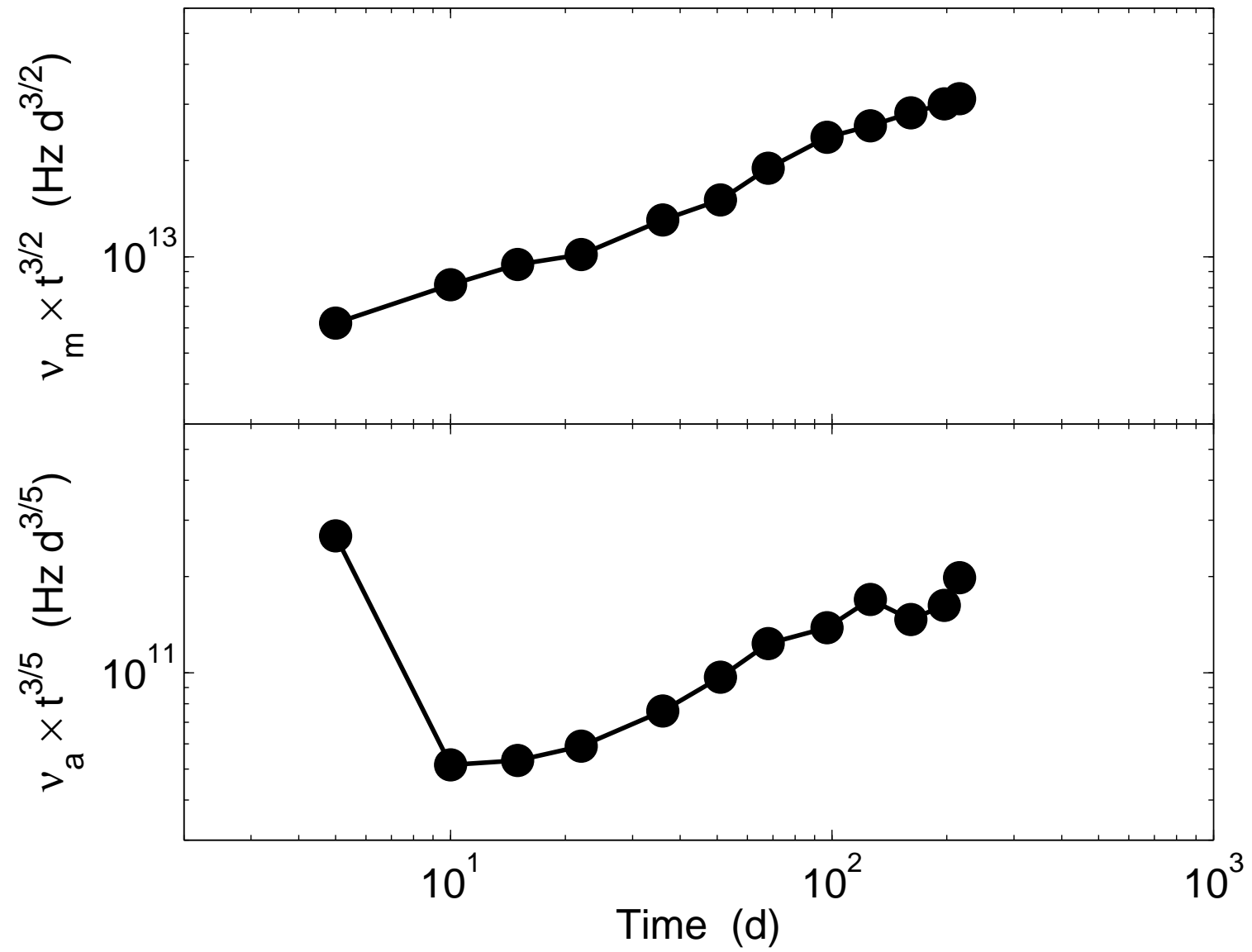

FIG. 4. - Temporal evolution of the synchrotron frequencies $\nu_{m}$ (top) and $\nu_{a}$ (bottom) relative to the expected evolution in a simple model with a constant energy and a Wind profile. The shallower decline of $\nu_{m} \propto E_{j}^{0.5}$, with a particularly shallow evolution at $\delta t \approx 30-100 \mathrm{~d}$, is indicative of a continuous increase in energy. Similarly, the shallower decline of $\nu_{a}$, followed by a rapid increase, is indicative of a density profile of $\rho \propto r^{-1.5}$ and a flattening at $\delta t \approx 30-100 \mathrm{~d}$. 


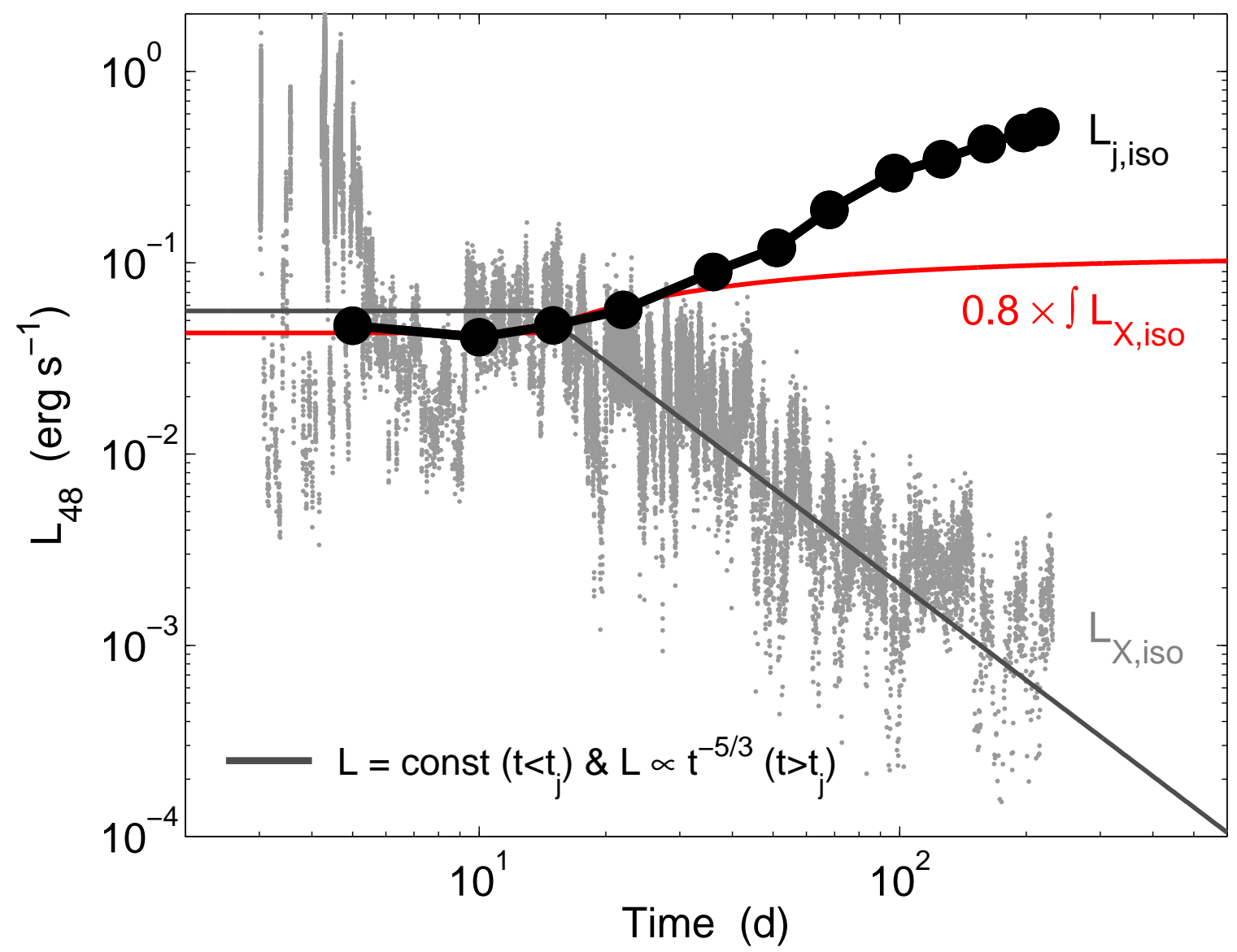

FIG. 5.- Temporal evolution of the integrated luminosity (or alternatively $E_{\mathrm{j}, \text { iso }}=L_{\mathrm{j}, \text { iso }} t_{j}$; black circles) in comparison to the X-ray luminosity (gray dots) as parametrized with a simple luminosity evolution (gray line). The red curve is the integrated luminosity derived from the simple model. The observed X-ray luminosity indicates that the fraction of total energy emitted in $X$-rays is comparable to the energy in the relativistic outflow (i.e., $\epsilon_{X} \approx 0.5$ ). The large increase in energy inferred from the radio observations cannot be explained by injection from a $L \propto t^{-5 / 3}$ tail. 


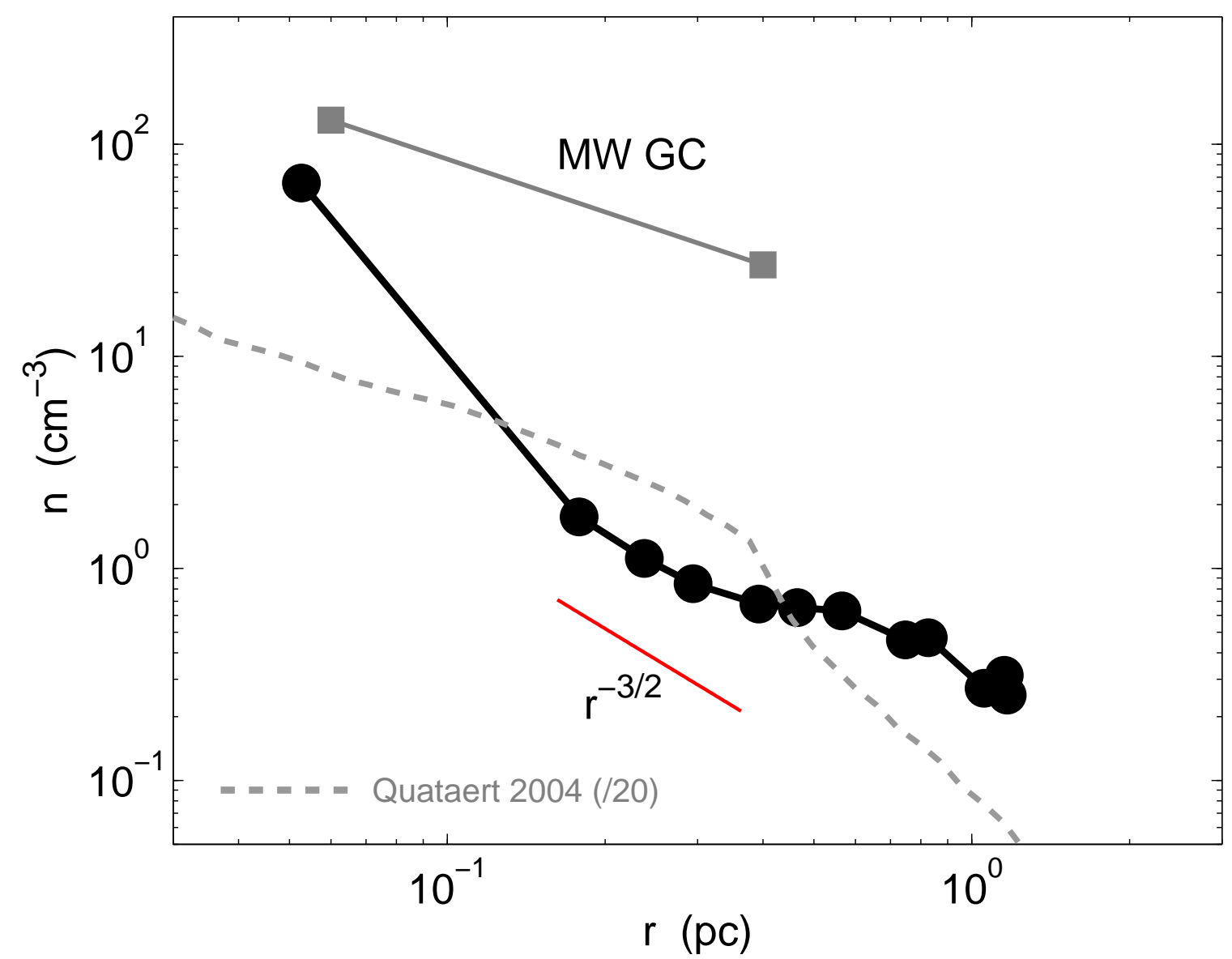

FIG. 6.- Radial density profile in the inner parsec around $\mathrm{Sw} 1644+57$ as inferred from the radio observations (black circles). The overall profile follows $\rho \propto r^{-3 / 2}$, with a significant flattening at $r \approx 0.4-0.6 \mathrm{pc}$. Following the flattening, the profile appears to recover to $r^{-3 / 2}$ by about $1 \mathrm{pc}$. Also shown is the density inferred from X-ray observations of the Galactic center (gray squares; Baganoff et al. 2003), which is about a factor of 30 times larger at $\approx 0.5 \mathrm{pc}$. The dashed line is a scaled-down model of the Galactic center density profile assuming gas feeding from massive stars in which the bulk of the gas is thermally expelled in a wind Quataert 2004). In this model the inner profile $(\lesssim 0.2 \mathrm{pc})$ is $\propto r^{-3 / 2}$, while the outer profile $(\gtrsim 0.4 \mathrm{pc})$ has a Wind $\left(\propto r^{-2}\right)$ profile. 


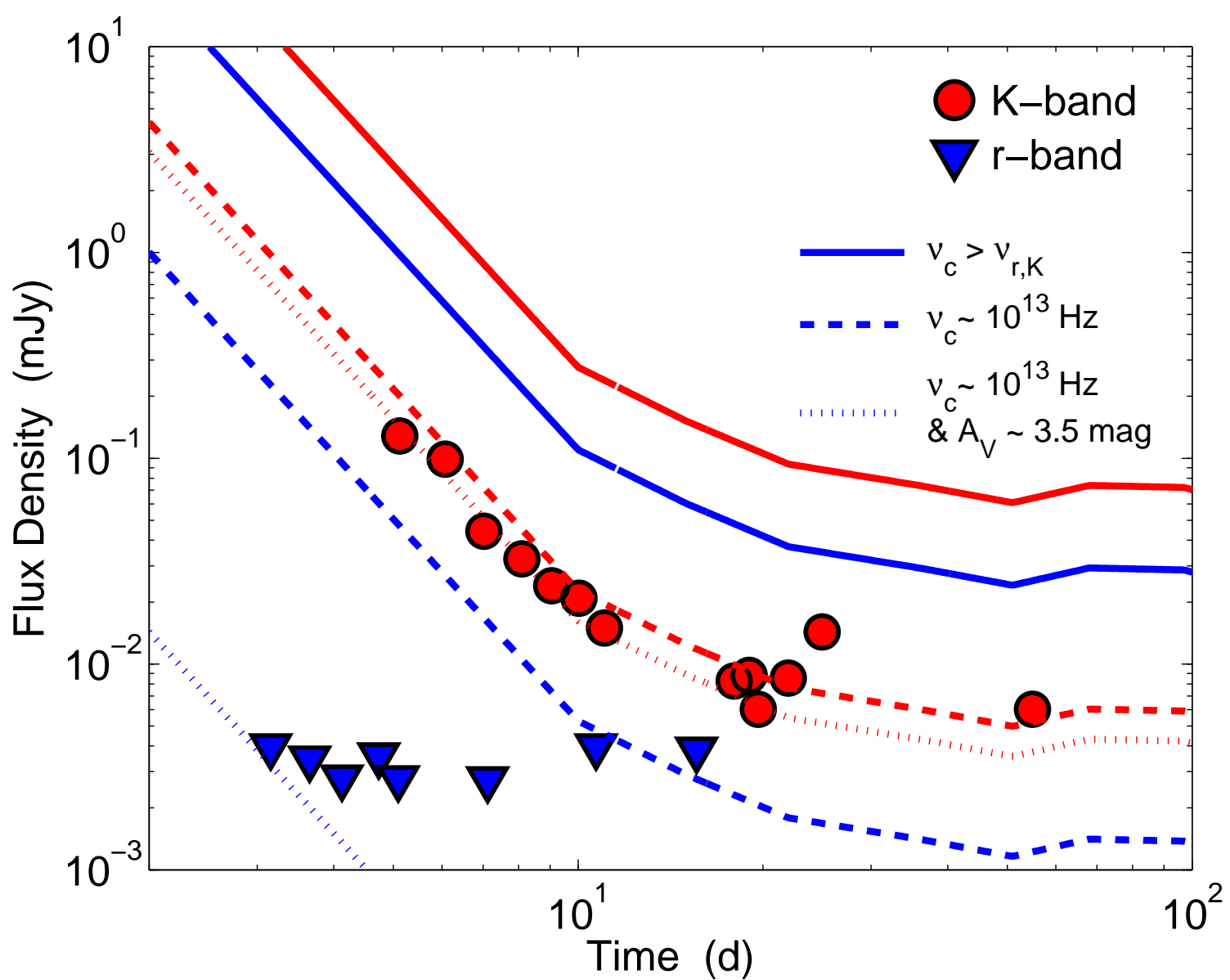

FIG. 7.- Predicted optical ( $r$-band; blue) and near-infrared ( $K$-band; red) light curves using the results of the radio modeling. The upper limits in $r$-band and detections in $K$-band are from Levan et al. (2011). Since the $K$-band fluxes are the total for Sw $1644+57$ and its host galaxy we have subtracted an estimated host contribution of about $20 \mu \mathrm{Jy}(K \approx 20.6 \mathrm{AB}$ mag). The solid lines are models without a cooling break between the radio and optical/near-IR, which clearly over-estimate the $K$-band flux. The dashed lines include a cooling break at $\nu_{c} \approx 10^{13} \mathrm{~Hz}$, and the dotted lines add host galaxy extinction of $A_{V} \approx 3.5 \mathrm{mag}$ to account for the optical non-detections. The combination of a cooling break and extinction provides an excellent fit to the near-IR evolution. 


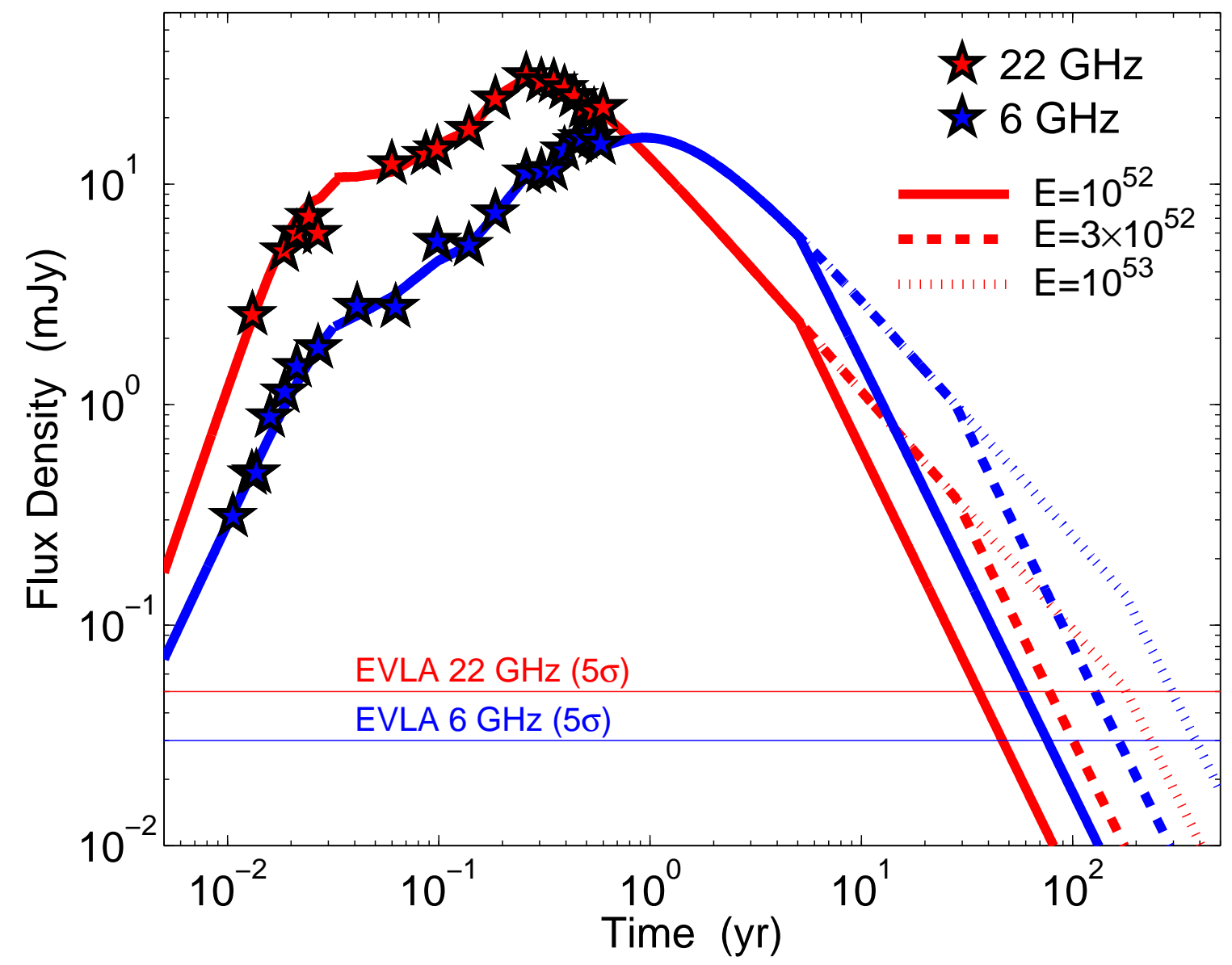

FIG. 8. - Predicted evolution of the radio light curves at $6 \mathrm{GHz}$ (blue) and $22 \mathrm{GHz}$ (red) assuming a radial density profile of $\rho \propto r^{-1.5}$ at $r \gtrsim 1$ pc and three values for the maximum integrated beaming-corrected energy (solid: $E_{j}=10^{52} \mathrm{erg}$; dashed: $E_{j}=3 \times 10^{52} \mathrm{erg}$; dotted: $E_{j}=10^{53} \mathrm{erg}$ ). The thin horizontal lines mark the $5 \sigma$ sensitivity of the EVLA, and indicate that the radio emission from Sw 1644+57 should be detectable for decades (and perhaps centuries) at centimeter wavelengths. 


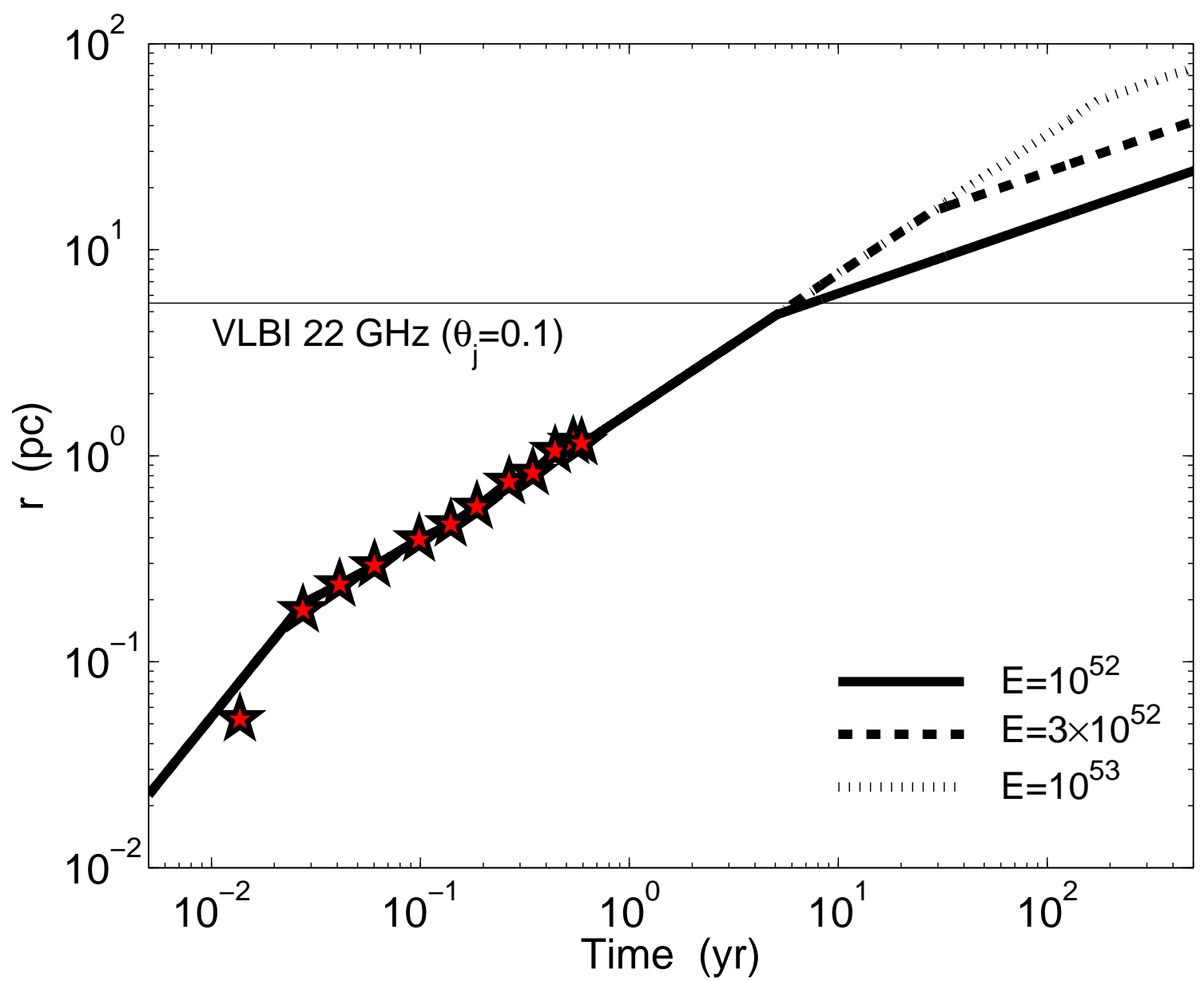

FIG. 9.- Predicted evolution of the jet radius assuming a radial density profile of $\rho \propto r^{-1.5}$ at $r \gtrsim 1 \mathrm{pc}$ and three values for the maximum beaming energy (solid: $E_{j}=10^{52} \mathrm{erg}$; dashed: $E_{j}=3 \times 10^{52} \mathrm{erg}$; dotted: $E_{j}=10^{53} \mathrm{erg}$ ). The thin horizontal line marks the resolution of VLBI for a jet opening angle of $\theta_{j}=0.1$. The source should become resolvable at $\delta t \sim 6 \mathrm{yr}$, with an expected $22 \mathrm{GHz}$ flux density of about $2 \mathrm{mJy}$ (Figure 8 . If the jet instead begins to undergo significant spreading it may become resolvable at $\sim 1$ yr when the $22 \mathrm{GHz}$ flux density is still $\sim 10 \mathrm{mJy}$. 